\title{
Renormalized and Entropy Solutions of Tumor Growth Model with Nonlinear Acid Production
}

\section{Shangerganesh Lingeshwaran ${ }^{a}$, Deiva Mani Nallasamy ${ }^{b}$ and Karthikeyan Shanmugasundaram ${ }^{b}$}

${ }^{a}$ Department of Humanities and Sciences

National Institute of Technology Goa, Farmagudi, Goa, India

${ }^{b}$ Department of Mathematics

Periyar University, Salem, Tamilnadu, India

E-mail(corresp.): shangerganesh@gmail.com

E-mail: shangerganesh@nitgoa.ac.in

E-mail: deivamani.vn@gmail.com

E-mail: skkmathpu@gmail.com

Received March 22, 2017; revised July 12, 2017; published online September 15, 2017

\begin{abstract}
This paper establishes the existence of renormalized and entropy solutions for a system of nonlinear reaction-diffusion equations which describes the tumor growth along with acidification and interaction. Under the assumptions of $L^{1}$ data and no growth conditions with zero Dirichlet boundary conditions, we prove the existence of renormalized and entropy solutions for the considered mathematical model.

Keywords: acid-mediated tumor growth, renormalized solution, entropy solution.
\end{abstract}

AMS Subject Classification: 35K57; 35K65; 92D25; 35D99.

\section{Introduction}

Acid-mediated tumor invasion model confines a mechanism linking altered glucose metabolism with the ability of tumor cells to form invasive cancers. Glucose metabolism and increased glucose uptake observed in the majority of clinical cancers which are critical for development of the invasive phenotype. Tumor cells are resistance to acid induced toxicity which survive and proliferate in low pH micro environments, invade the damaged adjacent normal tissues. First, acidification of the tumor micro environment is shown by Gatenby et al. [15] and Martin et al. [17] to increase invasiveness and metastasis of cancer cells using mathematical model. Acidification induced by the result of glycolysis both in the presence of oxygen through Warburg effect and intermittent hypoxia which produce toxicity in the surrounding normal tissue provides the empty space for tumor cell proliferation and invasion. Gatenby and Gawlinski [14] used the acid-mediated invasion hypothesis in a reaction-diffusion 
framework which plays an important role in tumor progression. The mathematical representation of a reaction-diffusion system at the tissue scale in which many mathematical models have been developed to explore the relationships between tumor invasion, tissue acidity and cellular metabolism and energy requirements, see, for example, Bertuzzi et al. [6], Bianchini and Fasano [7], Ganesan and Lingeshwaran [13], McGillen et al. [19], Smallbone et al. [26], Tao et al. [27] and Venkatasubramanian et al. [28].

Acidification factor plays a major role in the development of tumor growth models. Therefore, in this work, we consider a PDE model for acid-mediated tumor growth extended from [14] with nonlinear acidification term to capture a wider range of tumor behaviors. Here, we also have included the interacting phenomena, that is, as tumor cell density increases with time, metabolism which produces $\mathrm{H}^{+}$ions leads to destruction of normal cells surrounding the tumor and thus a reduction in normal cell density. Thus, the nonlinear reactiondiffusion mathematical model is given by

$$
\left\{\begin{array}{l}
\frac{\partial u_{1}}{\partial t}-\operatorname{div}\left(\sigma_{1}\left(u_{2}\right) d_{1}\left(u_{1}, \nabla u_{1}\right)\right)=r_{1}\left(\rho_{1}\left(u_{1}\right)-u_{2} \mu_{1}\left(u_{1}\right)\right) \\
-\pi\left(u_{1}, u_{3}\right)+f(x, t) \text { in } Q_{T} \\
\frac{\partial u_{2}}{\partial t}-\operatorname{div}\left(\sigma_{2}\left(u_{1}\right) d_{2}\left(u_{2}, \nabla u_{2}\right)\right)=r_{2}\left(\rho_{2}\left(u_{2}\right)-u_{1} \mu_{2}\left(u_{2}\right)\right)+g(x, t) \text { in } Q_{T} \\
\frac{\partial u_{3}}{\partial t}-\sigma_{3} \operatorname{div}\left(d_{3}\left(u_{3}, \nabla u_{3}\right)\right)=r_{3} \rho_{2}\left(u_{2}\right)-\alpha_{3} u_{3}+h(x, t) \text { in } Q_{T}
\end{array}\right.
$$

with initial and boundary conditions

$$
\begin{array}{ll}
u_{i}(x, 0)=u_{i, 0}(x), \quad i=1,2,3 & \text { in } \Omega \\
u_{i}(x, t)=0, \quad i=1,2,3 & \text { on } \Sigma_{T}
\end{array}
$$

where $Q_{T}=\Omega \times(0, T), \Sigma_{T}=\partial \Omega \times(0, T), \Omega$ is an open bounded domain in $\mathbb{R}^{N}$ with boundary $\partial \Omega$ (no smoothness is assumed on the boundary $\partial \Omega$ ). The mathematical model consists of three unknown variables: normal cell density $u_{1}(x, t)$, tumor cell density $u_{2}(x, t)$ and acid concentration $u_{3}(x, t)$. The homogeneous Dirichlet boundary condition means that the model (1.1) is selfcontained and has no population on the boundary $\partial \Omega$. The density dependent diffusion coefficients for the normal cells and tumor cells are given by $\sigma_{1}\left(u_{2}\right)$ and $\sigma_{2}\left(u_{1}\right)$ respectively. Further, the excess $H^{+}$ions diffuse chemically with constant diffusion rate with $\sigma_{3}$. The normal cells and tumor cells obey the logistic growth $\rho_{1}\left(u_{1}\right)=u_{1}\left(1-\frac{u_{1}}{K_{1}}\right), \rho_{2}\left(u_{2}\right)=u_{2}\left(1-\frac{u_{2}}{K_{2}}\right)$ with growth rates $r_{1}$ and $r_{2}$ and carrying capacities $K_{1}$ and $K_{2}$. The competition relationship between the normal cells and tumor cells with the rates $a_{1}$ and $a_{2}$ are given by $\mu_{1}\left(u_{1}\right)=\frac{a_{1} u_{1}}{K_{1}}$ and $\mu_{2}\left(u_{2}\right)=\frac{a_{2} u_{2}}{K_{2}}$. The interaction of healthy cells with the excess $H^{+}$ions is given by $\pi\left(u_{1}, u_{3}\right)=\alpha_{1} u_{1} u_{3}$ which leads to a death rate proportional to the concentration of $\mathrm{H}^{+}$ions and denote the constant of proportionality by $\alpha_{1}$. Acidification caused at a rate $r_{3}$ proportional to the tumor cell density and an uptake term with constant proportionality $\alpha_{3}$ is included to measure the mechanisms for increasing extracellular $\mathrm{pH}$. Moreover, $f(x, t), g(x, t)$ and $h(x, t)$ denote the source terms of the respective equations. Further, we have assumed that all the coefficients are positive and $\sigma_{i}(\cdot) \in \mathbb{C}^{2}(\mathbb{R}), i=1,2$. 
The notion of renormalized solution is introduced by DiPerna and Lions for Boltzmann equations in [11]. Further, the same framework has extended for elliptic equations, parabolic equations and conservation laws, for example, see $[5,8,9,10,18,22,23]$ and also see the references therein. Furthermore, a new class of study, equivalence between renormalized and entropy solutions studied for parabolic equations by many researchers, for example, see [1,3,12]. Moreover, in the literature, considerable amount of works are available for the existence and uniqueness of biological models using various mathematical techniques, for example, see, $[2,4,24,25]$ and the references therein. As far as, acid-mediated cancer invasion model is concerned, only few papers available in the literature. Local and global existence of solutions of the model governed by acid-mediated tumor invasion established in [20]. Acid-mediated invasion model for tumor-stromal interactions under no flux boundary condition is concerned in [16] and the global existence and uniqueness proved using contraction mapping principle. A mathematical model focusing on the effect of heat shock proteins on the tumor cell migration is proposed and the local existence of a unique positive solution is obtained in [21]. On the otherhand, existence of renormalized and entropy solutions for the system of parabolic equations concerned, only few papers available in the literature, see, [3,4,24,25]. Therefore, in contrast to the above mentioned papers, in this work, the main novel point is to establish the existence of renormalized and entropy solutions of the model governed by the acid-mediated tumor growth under no growth conditions and integrable data.

The paper is organized as follows. In Section 2, we state the main theorem, that is, existence of renormalized solution of the parabolic system (1.1). Then, we introduce the regularized system of (1.1) and establish the existence of weak solutions of the regularized system. Further, we prove the existence of renormalized solution of (1.1) using the lemmas established in that section. Finally, in Section 3, we prove that renormalized solution of (1.1) is also an entropy solution.

\section{Renormalized solutions for cancer invasion system}

In this section, first we define the renormalized solution for the given parabolic system (1.1). After stating the main result of the work, we introduce regularized system for (1.1) and then we establish the existence of weak solutions of the regularized problem using the Faedo-Galerkin approximation method. Furthermore, we state and prove the certain lemmas which are useful to prove the existence of renormalized solutions of (1.1).

Definition 1. A renormalized solution of (1.1) is a set of functions $\left(u_{1}, u_{2}, u_{3}\right)$ satisfying the following conditions: $u_{1}(x, t), u_{2}(x, t)$ and $u_{3}(x, t)$ are all nonnegative for a.e. $(x, t) \in Q_{T}$. For $i=1,2,3$

$$
\begin{aligned}
& u_{i} \in L^{\infty}\left(0, T ; L^{1}(\Omega)\right) \cap L^{2}\left(0, T ; H_{0}^{1}(\Omega)\right), \quad T_{k}\left(u_{i}\right) \in L^{2}\left(0, T ; H_{0}^{1}(\Omega)\right) \\
& \text { for any } k \geq 0, \quad \int_{\left(n \leq\left|u_{i}\right| \leq n+1\right)} d_{i}\left(u_{i}, \nabla u_{i}\right) \nabla u_{i} \mathrm{~d} x \mathrm{~d} t \rightarrow 0 \quad \text { as } n \rightarrow \infty
\end{aligned}
$$


For all $S\left(u_{i}\right) \in C^{\infty}(\mathbb{R})$ with supp $S^{\prime}$ is compact,

$$
\begin{aligned}
& \frac{\partial S\left(u_{1}\right)}{\partial t}-\operatorname{div}\left(\sigma_{1}\left(u_{2}\right) S^{\prime}\left(u_{1}\right) d_{1}\left(u_{1}, \nabla u_{1}\right)\right)+\sigma_{1}\left(u_{1}\right) d_{1}\left(u_{1}, \nabla u_{1}\right) S^{\prime \prime}\left(u_{1}\right) \nabla u_{1} \\
& =r_{1} \rho_{1}\left(u_{1}\right) S^{\prime}\left(u_{1}\right)-r_{1} u_{2} \mu_{1}\left(u_{1}\right) S^{\prime}\left(u_{1}\right)-\pi\left(u_{1}, u_{3}\right) S^{\prime}\left(u_{1}\right)+f S^{\prime}\left(u_{1}\right) \operatorname{in} \mathcal{D}^{\prime}\left(Q_{T}\right), \\
& \frac{\partial S\left(u_{2}\right)}{\partial t}-\operatorname{div}\left(\sigma_{2}\left(u_{1}\right) S^{\prime}\left(u_{2}\right) d_{2}\left(u_{2}, \nabla u_{2}\right)\right)+\sigma_{2}\left(u_{1}\right) d_{2}\left(u_{2}, \nabla u_{2}\right) S^{\prime \prime}\left(u_{2}\right) \nabla u_{2} \\
& \quad=r_{2} \rho_{2}\left(u_{2}\right) S^{\prime}\left(u_{2}\right)-r_{2} u_{1} \mu_{2}\left(u_{2}\right) S^{\prime}\left(u_{2}\right)+g S^{\prime}\left(u_{2}\right) \quad \text { in } \mathcal{D}^{\prime}\left(Q_{T}\right), \\
& \frac{\partial S\left(u_{3}\right)}{\partial t}-\sigma_{3} \operatorname{div}\left(S^{\prime}\left(u_{3}\right) d_{3}\left(u_{3}, \nabla u_{3}\right)\right)+\sigma_{3} d_{3}\left(u_{3}, \nabla u_{3}\right) S^{\prime \prime}\left(u_{3}\right) \nabla u_{3} \\
& \quad=r_{3} \rho_{2}\left(u_{2}\right) S^{\prime}\left(u_{3}\right)-\alpha_{3} u_{3} S^{\prime}\left(u_{3}\right)+h S^{\prime}\left(u_{3}\right) \quad \text { in } \mathcal{D}^{\prime}\left(Q_{T}\right)
\end{aligned}
$$

and the initial conditions

$$
S\left(u_{i}(x, 0)\right)=S\left(u_{i, 0}(x)\right) \text { in } \Omega .
$$

Here, $\mathcal{D}\left(Q_{T}\right)$ denotes the set of all infinitely differentiable functions on $Q_{T}$ with compact support and the distributions on $Q_{T}$ are denoted by $\mathcal{D}^{\prime}\left(Q_{T}\right)$. We assume the following hypotheses to establish the existence of weak-renormalized solutions of the system (1.1), as in $[8,9]$ :

(H1) $\quad d_{i}(\eta, \zeta) \zeta \geq \gamma_{i}|\zeta|^{2}$, for every $\zeta \in \mathbb{R}^{N}$ and $\gamma_{i}>0, i=1,2,3$ is given real number.

(H2) For any $k>0$, there exists $C_{k}>0$ such that $\left|d_{1}(\eta, \zeta)\right| \leq C_{k}(1+|\zeta|)$.

(H4) $u_{i, 0}(x), \in L^{1}(\Omega), i=1,2,3$.

(H5) $f, g, h \in L^{1}\left(Q_{T}\right)$.

Further, we introduce the truncation function at the height $k$,

$$
\begin{aligned}
& T_{k}(z)= \begin{cases}k, & \text { if } \quad z \geq k, \\
z, & \text { if }|z| \leq k, \\
-k, & \text { if } z \leq-k,\end{cases} \\
& \widetilde{T}_{k}(z)=\int_{0}^{z} T_{k}(s) \mathrm{d} s= \begin{cases}z^{2} / 2, & \text { if }|z| \leq k, \\
k|z|-k^{2} / 2, & \text { if } \quad|z| \geq k .\end{cases}
\end{aligned}
$$

Moreover, throughout this work, we use a generic constant $C$ instead of different constants.

Theorem 1. Under the hypotheses $(H 1)-(H 5)$, there exists at least one renormalized solution of the system (1.1) in the sense of Definition 1.

For $\varepsilon>0$, let us introduce the following approximations of the data:

(H6) $\quad u_{i, 0}^{\varepsilon} \in L^{2}(\Omega)$, and $u_{i, 0}^{\varepsilon} \rightarrow u_{i, 0}, i=1,2,3$ a.e. in $\Omega$ and strongly in $L^{1}(\Omega)$ as $\varepsilon$ tends to zero. 
(H7) $f^{\varepsilon}, g^{\varepsilon}, h^{\varepsilon} \in L^{2}\left(Q_{T}\right)$ and $f^{\varepsilon} \rightarrow f, g^{\varepsilon} \rightarrow g, h^{\varepsilon} \rightarrow h$ a.e. in $Q_{T}$ and strongly in $L^{1}\left(Q_{T}\right)$ as $\varepsilon$ tends to zero.

Consider the following approximation problem of the system (1.1) for $\varepsilon>0$ :

$$
\left\{\begin{array}{c}
\frac{\partial u_{1}^{\varepsilon}}{\partial t}-\operatorname{div}\left(\sigma_{1}\left(u_{2}^{\varepsilon}\right) d_{1}\left(u_{1}^{\varepsilon}, \nabla u_{1}^{\varepsilon}\right)\right)=r_{1} \rho_{1}^{\varepsilon}\left(u_{1}^{\varepsilon}\right)-r_{1} u_{2}^{\varepsilon} \mu_{1}^{\varepsilon}\left(u_{1}^{\varepsilon}\right) \\
-\pi^{\varepsilon}\left(u_{1}^{\varepsilon}, u_{3}^{\varepsilon}\right)+f^{\varepsilon} \text { in } Q_{T} \\
\frac{\partial u_{2}^{\varepsilon}}{\partial t}-\operatorname{div}\left(\sigma_{2}\left(u_{1}^{\varepsilon}\right) d_{2}\left(u_{2}^{\varepsilon}, \nabla u_{2}^{\varepsilon}\right)\right)=r_{2} \rho_{2}^{\varepsilon}\left(u_{2}^{\varepsilon}\right)-r_{2} u_{1}^{\varepsilon} \mu_{2}^{\varepsilon}\left(u_{2}^{\varepsilon}\right)+g^{\varepsilon} \operatorname{in} Q_{T} \\
\frac{\partial u_{3}^{\varepsilon}}{\partial t}-\sigma_{3} \operatorname{div}\left(d_{3}\left(u_{3}^{\varepsilon}, \nabla u_{3}^{\varepsilon}\right)\right)=r_{3} \rho_{2}^{\varepsilon}\left(u_{2}^{\varepsilon}\right)-\alpha_{3} u_{3}^{\varepsilon}+h^{\varepsilon} \text { in } Q_{T}
\end{array}\right.
$$

with initial and boundary conditions

$$
\begin{array}{ll}
u_{i}^{\varepsilon}(x, 0)=u_{i, 0}^{\varepsilon}(x), \quad i=1,2,3 & \text { in } \Omega, \\
u_{i}^{\varepsilon}(x, t)=0, \quad i=1,2,3 & \text { on } \Sigma_{T},
\end{array}
$$

where $\rho_{i}^{\varepsilon}\left(u_{i}\right)=\rho_{i}\left(u_{i}^{\varepsilon}\right) /\left(1+\varepsilon \rho_{i}\left(u_{i}^{\varepsilon}\right)\right), \mu_{i}^{\varepsilon}\left(u_{i}^{\varepsilon}\right)=\mu\left(u_{i}^{\varepsilon}\right) /\left(1+\varepsilon \mu\left(u_{i}^{\varepsilon}\right)\right)$, for $i=1,2$ and $\pi^{\varepsilon}\left(u_{1}^{\varepsilon}, u_{3}^{\varepsilon}\right)=\pi\left(u_{1}^{\varepsilon}, u_{3}^{\varepsilon}\right) /\left(1+\varepsilon \pi\left(u_{1}^{\varepsilon}, u_{3}^{\varepsilon}\right)\right)$.

Lemma 1. Under the hypotheses $(H 1-H 3)$ and $(H 6-H 7)$ the approximation system (2.1) admits unique weak solution

$$
u_{i}^{\varepsilon} \in L^{2}\left(0, T ; H_{0}^{1}(\Omega)\right) \cap L^{\infty}\left(0, T ; L^{2}(\Omega)\right) \cap C\left([0, T], L^{2}(\Omega)\right),
$$

with $\frac{\partial u_{i}^{\varepsilon}}{\partial t} \in L^{2}\left(0, T ; H^{-1}(\Omega)\right)$ such that for any $\phi_{i} \in L^{2}\left(0, T ; H_{0}^{1}(\Omega)\right), i=1,2,3$,

$$
\begin{aligned}
& \int_{0}^{T}\left\langle\partial_{t} u_{1}^{\varepsilon}, \phi_{1}\right\rangle \mathrm{d} t+\int_{Q_{T}} \sigma_{1}\left(u_{2}^{\varepsilon}\right) d_{1}\left(u_{1}^{\varepsilon}, \nabla u_{1}^{\varepsilon}\right) \nabla \phi_{1} \mathrm{~d} x \mathrm{~d} t=r_{1} \int_{Q_{T}} \rho_{1}^{\varepsilon}\left(u_{1}^{\varepsilon}\right) \phi_{1} \mathrm{~d} x \mathrm{~d} t \\
& \quad-r_{1} \int_{Q_{T}} u_{2}^{\varepsilon} \mu_{1}^{\varepsilon}\left(u_{1}^{\varepsilon}\right) \phi_{1} \mathrm{~d} x \mathrm{~d} t-\int_{Q_{T}} \pi^{\varepsilon}\left(u_{1}^{\varepsilon}, u_{3}^{\varepsilon}\right) \phi_{1} \mathrm{~d} x \mathrm{~d} t+\int_{Q_{T}} f^{\varepsilon} \phi_{1} \mathrm{~d} x \mathrm{~d} t \\
& \int_{0}^{T}\left\langle\partial_{t} u_{2}^{\varepsilon}, \phi_{2}\right\rangle \mathrm{d} t+\int_{Q_{T}} \sigma_{2}\left(u_{1}^{\varepsilon}\right) d_{2}\left(u_{2}^{\varepsilon}, \nabla u_{2}^{\varepsilon}\right) \nabla \phi_{2} \mathrm{~d} x \mathrm{~d} t \\
& \quad=r_{2} \int_{Q_{T}} \rho_{2}^{\varepsilon}\left(u_{2}^{\varepsilon}\right) \phi_{2} \mathrm{~d} x \mathrm{~d} t-r_{2} \int_{Q_{T}} u_{1}^{\varepsilon} \mu_{2}^{\varepsilon}\left(u_{2}^{\varepsilon}\right) \phi_{2} \mathrm{~d} x \mathrm{~d} t+\int_{Q_{T}} g^{\varepsilon} \phi_{2} \mathrm{~d} x \mathrm{~d} t \\
& \int_{0}^{T}\left\langle\partial_{t} u_{3}^{\varepsilon}, \phi_{3}\right\rangle \mathrm{d} t+\sigma_{3} \int_{Q_{T}} d_{3}\left(u_{3}^{\varepsilon}, \nabla u_{3}^{\varepsilon}\right) \nabla \phi_{3} \mathrm{~d} x \mathrm{~d} t \\
& =r_{3} \int_{Q_{T}} \rho_{2}^{\varepsilon}\left(u_{2}^{\varepsilon}\right) \phi_{3} \mathrm{~d} x \mathrm{~d} t-\alpha_{3} \int_{Q_{T}} u_{3}^{\varepsilon} \phi_{3} \mathrm{~d} x \mathrm{~d} t+\int_{Q_{T}} h^{\varepsilon} \phi_{3} \mathrm{~d} x \mathrm{~d} t
\end{aligned}
$$

hold.

Proof. To use the Faedo-Galerkin approximation method, let us consider an appropriate spectral problem, see [4], in which the corresponding eigenfunctions $e_{l}(x)$ form an orthogonal basis in $H_{0}^{1}(\Omega)$ and orthonormal basis in $L^{2}(\Omega)$. Our aim is to identify the finite dimensional approximation solutions for the system (2.1) as sequences $\left\{u_{i, n}^{\varepsilon}\right\}, i=1,2,3$ defined for $n \geq 1, t \geq 0$ and $x \in \bar{\Omega}$ by

$$
u_{i, n}^{\varepsilon}(x, t)=\sum_{l=1}^{n} c_{i, n, l}(t) e_{l}(x), \quad i=1,2,3
$$


with the initial conditions

$$
u_{i, n}^{\varepsilon}(x, 0)=u_{i, 0, n}(x):=\sum_{l=1}^{n} c_{i, n, l}(0) e_{l}(x) .
$$

Further, it should be remarked that the above form of solutions should satisfy the required boundary conditions. Next, we have to determine the set of coefficients $\left\{c_{i, n, l}\right\}_{l=1}^{n}, i=1,2,3$ such that, for $m=1,2, \ldots, n$

$$
\left\{\begin{array}{c}
\int_{\Omega} \partial_{t} u_{1, n}^{\varepsilon} e_{m} \mathrm{~d} x+\int_{\Omega} \sigma_{1}\left(u_{2, n}^{\varepsilon}\right) d_{1}\left(u_{1, n}^{\varepsilon}, \nabla u_{1, n}^{\varepsilon}\right) \nabla e_{m} \mathrm{~d} x=r_{1} \int_{\Omega} \rho_{1}^{\varepsilon}\left(u_{1, n}^{\varepsilon}\right) e_{m} \mathrm{~d} x \\
-r_{1} \int_{\Omega} u_{2, n}^{\varepsilon} \mu_{1}^{\varepsilon}\left(u_{1, n}^{\varepsilon}\right) e_{m} \mathrm{~d} x-\int_{\Omega} \pi^{\varepsilon}\left(u_{1, n}^{\varepsilon}, u_{3, n}^{\varepsilon}\right) e_{m} \mathrm{~d} x+\int_{\Omega} f^{\varepsilon} e_{m} \mathrm{~d} x \\
\int_{\Omega} \partial_{t} u_{2, n}^{\varepsilon} e_{m} \mathrm{~d} x+\int_{\Omega} \sigma_{2}\left(u_{1, n}^{\varepsilon}\right) d_{2}\left(u_{2, n}^{\varepsilon}, \nabla u_{2, n}^{\varepsilon}\right) \nabla e_{m} \mathrm{~d} x \\
=r_{2} \int_{\Omega} \rho_{2}^{\varepsilon}\left(u_{2, n}^{\varepsilon}\right) e_{m} \mathrm{~d} x-r_{2} \int_{\Omega} u_{1, n}^{\varepsilon} \mu_{2}^{\varepsilon}\left(u_{2, n}^{\varepsilon}\right) e_{m} \mathrm{~d} x+\int_{\Omega} g^{\varepsilon} e_{m} \mathrm{~d} x \\
\int_{\Omega} \partial_{t} u_{3, n}^{\varepsilon} e_{m} \mathrm{~d} x+\sigma_{3} \int_{\Omega} d_{3}\left(u_{3, n}^{\varepsilon}, \nabla u_{3, n}^{\varepsilon}\right) \nabla e_{m} \mathrm{~d} x \\
=r_{3} \int_{\Omega} \rho_{2}^{\varepsilon}\left(u_{2, n}^{\varepsilon}\right) e_{m} \mathrm{~d} x-\alpha_{3} \int_{\Omega} u_{3, n}^{\varepsilon} e_{m} \mathrm{~d} x+\int_{\Omega} h^{\varepsilon} e_{m} \mathrm{~d} x
\end{array}\right.
$$

Now (2.2) can be rewritten in the following form:

$$
\left\{\begin{array}{c}
c_{1, n, m}^{\prime}(t)=-\int_{\Omega} \sigma_{1}\left(u_{2, n}^{\varepsilon}\right) d_{1}\left(u_{1, n}^{\varepsilon}, \nabla u_{1, n}^{\varepsilon}\right) \nabla e_{m} \mathrm{~d} x+r_{1} \int_{\Omega} \rho_{1}^{\varepsilon}\left(u_{1, n}^{\varepsilon}\right) e_{m} \mathrm{~d} x \\
-r_{1} \int_{\Omega} u_{2, n}^{\varepsilon} \mu_{1}^{\varepsilon}\left(u_{1, n}^{\varepsilon}\right) e_{m} \mathrm{~d} x-\int_{\Omega} \pi^{\varepsilon}\left(u_{1, n}^{\varepsilon}, u_{3, n}^{\varepsilon}\right) e_{m} \mathrm{~d} x+\int_{\Omega} f^{\varepsilon} e_{m} \mathrm{~d} x \\
=: G_{1}^{m}\left(t,\left\{c_{1, n, l}\right\}_{l=1}^{n},\left\{c_{2, n, l}\right\}_{l=1}^{n},\left\{c_{3, n, l}\right\}_{l=1}^{n}\right), \\
c_{2, n, m}^{\prime}(t)=-\int_{\Omega} \sigma_{2}\left(u_{1, n}^{\varepsilon}\right) d_{2}\left(u_{2, n}^{\varepsilon}, \nabla u_{2, n}^{\varepsilon}\right) \nabla e_{m} \mathrm{~d} x+r_{2} \int_{\Omega} \rho_{2}\left(u_{2, n}^{\varepsilon}\right) e_{m} \mathrm{~d} x \\
-r_{2} \int_{\Omega} u_{1, n}^{\varepsilon} \mu_{2}^{\varepsilon}\left(u_{2, n}^{\varepsilon}\right) e_{m} \mathrm{~d} x+\int_{\Omega} g^{\varepsilon} e_{m} \mathrm{~d} x \\
=: G_{2}^{m}\left(t,\left\{c_{1, n, l}\right\}_{l=1}^{n},\left\{c_{2, n, l}\right\}_{l=1}^{n}\right), \\
c_{3, n, m}^{\prime}(t)=-\sigma_{3} \int_{\Omega} d_{3}\left(u_{3, n}^{\varepsilon}, \nabla u_{3, n}^{\varepsilon}\right) \nabla e_{m} \mathrm{~d} x+r_{3} \int_{\Omega} \rho_{2}^{\varepsilon}\left(u_{2, n}^{\varepsilon}\right) e_{m} \mathrm{~d} x \\
-\alpha_{3} \int_{\Omega} u_{3, n}^{\varepsilon} e_{m} \mathrm{~d} x+\int_{\Omega} h^{\varepsilon} e_{m} \mathrm{~d} x=: G_{3}^{m}\left(t,\left\{c_{2, n, l}\right\}_{l=1}^{n},\left\{c_{3, n, l}\right\}_{l=1}^{n}\right) .
\end{array}\right.
$$

Let $\kappa \in(0, T)$ and set $U=[0, \kappa]$. Choose $R>0$ large enough so that the ball $B_{R} \subset \mathbb{R}^{N}$ contains $\left\{c_{i, n, l}(0)\right\}, i=1,2,3$ and set $V=\bar{B}_{R}$. The components $G_{i}^{m}, i=1,2,3$ can be bounded on $U \times V$ as follows:

$$
\begin{aligned}
& \left|G_{1}^{m}\left(t,\left\{c_{1, n, l}\right\}_{l=1}^{n},\left\{c_{2, n, l}\right\}_{l=1}^{n},\left\{c_{3, n, l}\right\}_{l=1}^{n}\right)\right| \leq\left\|\sigma_{1}\left(\sum_{l=1}^{n} c_{2, n, l}(t) e_{l}(x)\right)\right\|_{L^{\infty}(\Omega)} \\
& \times\left(\int_{\Omega}\left|d_{1}\left(\sum_{l=1}^{n} c_{1, n, l}(t) e_{l}(x), \sum_{l=1}^{n} c_{1, n, l}(t) \nabla e_{l}(x)\right)\right|^{2} \mathrm{~d} x\right)^{\frac{1}{2}}
\end{aligned}
$$




$$
\begin{aligned}
& \left(\int_{\Omega}\left|\nabla e_{m}\right|^{2} \mathrm{~d} x\right)^{\frac{1}{2}}+\frac{\left(r_{1}+1\right)}{\varepsilon} \operatorname{meas}(\Omega)\left(\int_{\Omega}\left|e_{m}\right|^{2} \mathrm{~d} x\right)^{\frac{1}{2}} \\
& \quad+\frac{r_{1}}{\varepsilon}\left(\int_{\Omega}\left|\sum_{l=1}^{n} c_{2, n, l}(t) e_{l}(x)\right|^{2} \mathrm{~d} x\right)^{\frac{1}{2}}\left(\int_{\Omega}\left|e_{m}\right|^{2} \mathrm{~d} x\right)^{\frac{1}{2}}+\int_{\Omega} f^{\varepsilon} e_{m} \mathrm{~d} x \\
& \quad \leq C(R, n),
\end{aligned}
$$

where the constant $C(R, n)>0$ depends only on $R$ and $n$. Similarly it is easy to obtain that

$$
\begin{aligned}
& \left|G_{2}^{m}\left(t,\left\{c_{1, n, l}\right\}_{l=1}^{n},\left\{c_{2, n, l}\right\}_{l=1}^{n}\right)\right| \leq C(R, n), \\
& \left|G_{3}^{m}\left(t,\left\{c_{2, n, l}\right\}_{l=1}^{n},\left\{c_{3, n, l}\right\}_{l=1}^{n}\right)\right| \leq C(R, n),
\end{aligned}
$$

where the constant $C(R, n)>0$ depends only on $R, n$.

According to the standard ODE theory, one can show that the absolutely continuous functions $\left\{c_{i, n, l}\right\}_{l=1}^{n}, i=1,2,3$ satisfy (2.3) and the initial conditions for $t \in\left[0, \kappa^{\prime}\right]$ a.e. where $\kappa^{\prime}>0$. Moreover, we have

$$
\begin{aligned}
& c_{1, n, l}(t)=c_{1, n, l}(0) \\
& \quad+\int_{0}^{t} G_{1}^{l}\left(\tau,\left\{c_{1, n, m}(\tau)\right\}_{m=1}^{n},\left\{c_{2, n, m}(\tau)\right\}_{m=1}^{n},\left\{c_{3, n, m}(\tau)\right\}_{m=1}^{n}\right) d \tau, \\
& c_{2, n, l}(t)=c_{2, n, l}(0)+\int_{0}^{t} G_{2}^{l}\left(\tau,\left\{c_{1, n, m}(\tau)\right\}_{m=1}^{n},\left\{c_{2, n, m}(\tau)\right\}_{m=1}^{n}\right) d \tau, \\
& c_{3, n, l}(t)=c_{3, n, l}(0)+\int_{0}^{t} G_{3}^{l}\left(\tau,\left\{c_{2, n, m}(\tau)\right\}_{m=1}^{n},\left\{c_{3, n, m}(\tau)\right\}_{m=1}^{n}\right) d \tau .
\end{aligned}
$$

This proves that the sequences are well defined and approximate solutions to the system $(2.3)$ on $\left[0, \kappa^{\prime}\right)$. Set

$$
\phi_{i, n}(x, t)=\sum_{l=1}^{n} b_{i, n, l}(t) e_{l}(x), \quad i=1,2,3,
$$

where the coefficients $b_{i, n, l}, i=1,2,3$ are absolutely continuous functions. Then from (2.2) the approximate solutions satisfy the weak formulation

$$
\left\{\begin{array}{c}
\int_{\Omega} \partial_{t} u_{1, n}^{\varepsilon} \phi_{1, n} \mathrm{~d} x+\int_{\Omega} \sigma_{1}\left(u_{2, n}^{\varepsilon}\right) d_{1}\left(u_{1, n}^{\varepsilon}, \nabla u_{1, n}^{\varepsilon}\right) \nabla \phi_{1, n} \mathrm{~d} x \\
=r_{1} \int_{\Omega} \rho_{1}^{\varepsilon}\left(u_{1, n}^{\varepsilon}\right) \phi_{1, n} \mathrm{~d} x-r_{1} \int_{\Omega} u_{2, n}^{\varepsilon} \mu_{1}^{\varepsilon}\left(u_{1, n}^{\varepsilon}\right) \phi_{1, n} \mathrm{~d} x \\
\quad-\int_{\Omega} \pi^{\varepsilon}\left(u_{1, n}^{\varepsilon}, u_{3, n}^{\varepsilon}\right) \phi_{1, n} \mathrm{~d} x+\int_{\Omega}^{f^{\varepsilon} \phi_{1, n} \mathrm{~d} x} \\
\int_{\Omega} \partial_{t} u_{2, n}^{\varepsilon} \phi_{2, n} \mathrm{~d} x+\int_{\Omega} \sigma_{2}\left(u_{1, n}^{\varepsilon}\right) d_{2}\left(u_{2, n}^{\varepsilon}, \nabla u_{2, n}^{\varepsilon}\right) \nabla \phi_{2, n} \mathrm{~d} x \\
=r_{2} \int_{\Omega} \rho_{2}^{\varepsilon}\left(u_{2, n}^{\varepsilon}\right) \phi_{2, n} \mathrm{~d} x-r_{2} \int_{\Omega} u_{1, n}^{\varepsilon} \mu_{2}^{\varepsilon}\left(u_{2, n}^{\varepsilon}\right) \phi_{2, n} \mathrm{~d} x+\int_{\Omega} g^{\varepsilon} \phi_{2, n} \mathrm{~d} x \\
\int_{\Omega} \partial_{t} u_{3, n}^{\varepsilon} \phi_{3, n} \mathrm{~d} x+\sigma_{3} \int_{\Omega} d_{3}\left(u_{3, n}^{\varepsilon}, \nabla u_{3, n}^{\varepsilon}\right) \nabla \phi_{3, n} \mathrm{~d} x \\
=r_{3} \int_{\Omega} \rho_{2}^{\varepsilon}\left(u_{2, n}^{\varepsilon}\right) \phi_{3, n} \mathrm{~d} x-\alpha_{3} \int_{\Omega} u_{3, n}^{\varepsilon} \phi_{3, n} \mathrm{~d} x+\int_{\Omega} h^{\varepsilon} \phi_{3, n} \mathrm{~d} x
\end{array}\right.
$$


Take $\phi_{i, n}=u_{i, n}^{\varepsilon}, i=1,2,3$ respectively in (2.4) and use Gronwall's lemma with Young's inequality to get

$$
\left\|u_{i, n}^{\varepsilon}\right\|_{L^{\infty}\left(0, \tilde{T} ; L^{2}(\Omega)\right)}+\left\|u_{i, n}^{\varepsilon}\right\|_{L^{2}\left(0, \tilde{T} ; H_{0}^{1}(\Omega)\right)} \leq C, \quad\left\|\partial_{t} u_{i, n}^{\varepsilon}\right\|_{L^{2}\left(0, \tilde{T} ; H^{-1}(\Omega)\right)} \leq C,
$$

where the constant $C>0$ and $\tilde{T}$ is an arbitrary time in the existence interval $\left[0, \kappa^{\prime}\right)$ of Galerkin solutions. Similar approach developed in $[2,4]$ is used to prove the global existence of solutions. Therefore, as $n \rightarrow \infty$, for $i=1,2,3$ we have,

$$
\begin{aligned}
u_{i, n}^{\varepsilon} & \rightarrow u_{i}^{\varepsilon} \text { weakly-* in } L^{\infty}\left(0, T ; L^{2}(\Omega)\right), \\
u_{i, n}^{\varepsilon} & \rightarrow u_{i}^{\varepsilon} \text { weakly in } L^{2}\left(0, T ; H_{0}^{1}(\Omega)\right), \\
d_{i}\left(u_{i, n}^{\varepsilon}, \nabla u_{i, n}^{\varepsilon}\right) & \rightarrow \eta_{i} \text { weakly in } L^{2}\left(Q_{T}\right), \\
\partial_{t} u_{i, n}^{\varepsilon} & \rightarrow \partial_{t} u_{i}^{\varepsilon} \text { weakly in } L^{2}\left(0, T ; H^{-1}(\Omega)\right) .
\end{aligned}
$$

Using similar type of arguments as in [4] we can show that $d_{i}\left(u_{i}^{\varepsilon}, \nabla u_{i}^{\varepsilon}\right)=\eta_{i}, i=$ $1,2,3$. Since the solutions $u_{1}^{\varepsilon}, u_{2}^{\varepsilon}, u_{3}^{\varepsilon} \in L^{2}\left(0, T ; H_{0}^{1}(\Omega)\right) \cap L^{\infty}\left(0, T ; L^{2}(\Omega)\right)$ and from the above a priori estimates we conclude that $u_{1}^{\varepsilon}, u_{2}^{\varepsilon}, u_{3}^{\varepsilon} \in C\left([0, T] ; L^{2}(\Omega)\right)$.

Lemma 2. Under the hypotheses $(H 6),(H 7)$ the functions $T_{k}\left(u_{i}^{\varepsilon}\right), \frac{\partial S\left(u_{i}^{\varepsilon}\right)}{\partial t}$, $i=1,2,3$, are bounded in $L^{2}\left(0, T ; H_{0}^{1}(\Omega)\right)$ and $L^{1}\left(Q_{T}\right)+L^{2}\left(0, T ; H^{-1}(\Omega)\right)$ respectively.

Proof. Taking $T_{k}\left(u_{1}^{\varepsilon}\right)$ as a test function in the first equation of (2.1) and integrating over $Q_{t}=\Omega \times(0, t)$, we get

$$
\begin{gathered}
\int_{Q_{t}} u_{1 s}^{\varepsilon} T_{k}\left(u_{1}^{\varepsilon}\right) \mathrm{d} x \mathrm{~d} s+\int_{Q_{t}} \sigma_{1}\left(u_{1}^{\varepsilon}\right) d_{1}\left(u_{1}^{\varepsilon}, \nabla u_{1}^{\varepsilon}\right) \nabla T_{k}\left(u_{1}^{\varepsilon}\right) \mathrm{d} x \mathrm{~d} s \\
=r_{1} \int_{Q_{t}} \rho_{1}^{\varepsilon}\left(u_{1}^{\varepsilon}\right) T_{k}\left(u_{1}^{\varepsilon}\right) \mathrm{d} x \mathrm{~d} s-r_{1} \int_{Q_{t}} u_{2}^{\varepsilon} \mu_{1}^{\varepsilon}\left(u_{1}^{\varepsilon}\right) T_{k}\left(u_{1}^{\varepsilon}\right) \mathrm{d} x \mathrm{~d} s \\
\quad-\int_{Q_{t}} \pi^{\varepsilon}\left(u_{1}^{\varepsilon}, u_{3}^{\varepsilon}\right) T_{k}\left(u_{1}^{\varepsilon}\right) \mathrm{d} x \mathrm{~d} s+\int_{Q_{t}} f^{\varepsilon} T_{k}\left(u_{1}^{\varepsilon}\right) \mathrm{d} x \mathrm{~d} s, \\
\int_{\Omega} \tilde{T}_{k}\left(u_{1}^{\varepsilon}\right)(t) \mathrm{d} x-\int_{\Omega} \widetilde{T}_{k}\left(u_{1,0}^{\varepsilon}\right) \mathrm{d} x+\gamma_{1} \int_{Q_{t}} \sigma_{1}\left(u_{2}^{\varepsilon}\right)\left|\nabla T_{k}\left(u_{1}^{\varepsilon}\right)\right|^{2} \mathrm{~d} x \mathrm{~d} s \\
\leq r_{1} \int_{Q_{t}} \rho_{1}^{\varepsilon}\left(u_{1}^{\varepsilon}\right) T_{k}\left(u_{1}^{\varepsilon}\right) \mathrm{d} x \mathrm{~d} s-r_{1} \int_{Q_{t}} u_{2}^{\varepsilon} \mu_{1}^{\varepsilon}\left(u_{1}^{\varepsilon}\right) T_{k}\left(u_{1}^{\varepsilon}\right) \mathrm{d} x \mathrm{~d} s \\
\quad-\int_{Q_{t}} \pi^{\varepsilon}\left(u_{1}^{\varepsilon}, u_{3}^{\varepsilon}\right) T_{k}\left(u_{1}^{\varepsilon}\right) \mathrm{d} x \mathrm{~d} s+\int_{Q_{t}} f^{\varepsilon} T_{k}\left(u_{1}^{\varepsilon}\right) \mathrm{d} x \mathrm{~d} s .
\end{gathered}
$$

Similarly, from the second and third equations of (2.1), we get

$$
\begin{aligned}
& \int_{\Omega} \tilde{T}_{k}\left(u_{2}^{\varepsilon}\right)(t) \mathrm{d} x-\int_{\Omega} \widetilde{T}_{k}\left(u_{2,0}^{\varepsilon}\right) \mathrm{d} x+\gamma_{2} \int_{Q_{t}} \sigma_{2}\left(u_{1}^{\varepsilon}\right)\left|\nabla T_{k}\left(u_{2}^{\varepsilon}\right)\right|^{2} \mathrm{~d} x \mathrm{~d} s \\
& \quad \leq r_{2} \int_{Q_{t}} \rho_{2}^{\varepsilon}\left(u_{2}^{\varepsilon}\right) T_{k}\left(u_{2}^{\varepsilon}\right) \mathrm{d} x \mathrm{~d} s-r_{2} \int_{Q_{t}} u_{1}^{\varepsilon} \mu_{2}^{\varepsilon}\left(u_{2}^{\varepsilon}\right) T_{k}\left(u_{2}^{\varepsilon}\right) \mathrm{d} x \mathrm{~d} s+\int_{Q_{t}} g^{\varepsilon} T_{k}\left(u_{2}^{\varepsilon}\right) \mathrm{d} x \mathrm{~d} s,
\end{aligned}
$$




$$
\begin{aligned}
& \int_{\Omega} \tilde{T}_{k}\left(u_{3}^{\varepsilon}\right)(t) \mathrm{d} x-\int_{\Omega} \widetilde{T}_{k}\left(u_{3,0}^{\varepsilon}\right) \mathrm{d} x+\sigma_{3} \gamma_{3} \int_{Q_{t}}\left|\nabla T_{k}\left(u_{3}^{\varepsilon}\right)\right|^{2} \mathrm{~d} x \mathrm{~d} s \\
& \leq r_{3} \int_{Q_{t}} \rho_{2}^{\varepsilon}\left(u_{2}^{\varepsilon}\right) T_{k}\left(u_{3}^{\varepsilon}\right) \mathrm{d} x \mathrm{~d} s-\alpha_{3} \int_{Q_{t}} u_{3}^{\varepsilon} T_{k}\left(u_{3}^{\varepsilon}\right) \mathrm{d} x \mathrm{~d} s+\int_{Q_{t}} h^{\varepsilon} T_{k}\left(u_{3}^{\varepsilon}\right) \mathrm{d} x \mathrm{~d} s .
\end{aligned}
$$

Add (2.5)-(2.8) and use Young's inequality, the properties of the functions $f^{\varepsilon}, g^{\varepsilon}, h^{\varepsilon}, u_{i, 0}^{\varepsilon}(x), i=1,2,3$, the continuity of $\sigma_{1}\left(u_{i}^{\varepsilon}\right), i=1,2$ and the boundedness of approximate solutions with the definition of the functions $\tilde{T}_{k}\left(u_{i}^{\varepsilon}\right), i=$ $1,2,3$ we have

$$
\int_{Q_{T}}\left|\nabla T_{k}\left(u_{i}^{\varepsilon}\right)\right|^{2} \mathrm{~d} x \mathrm{~d} s \leq C
$$

for any $C>0$ and $i=1,2,3$. From (2.9), we conclude that $T_{k}\left(u_{i}^{\varepsilon}\right), i=1,2,3$ is bounded in $L^{2}\left(0, T ; H_{0}^{1}(\Omega)\right)$. Multiplying first equation of $(2.1)$ by $S^{\prime}\left(u_{1}^{\varepsilon}\right)$, we get

$$
\begin{aligned}
& \frac{\partial S\left(u_{1}^{\varepsilon}\right)}{\partial t}=\operatorname{div}\left(S^{\prime}\left(u_{1}^{\varepsilon}\right) \sigma_{1}\left(u_{2}^{\varepsilon}\right) d_{1}\left(u_{1}^{\varepsilon}, \nabla u_{1}^{\varepsilon}\right)\right)-\sigma_{1}\left(u_{2}^{\varepsilon}\right) d_{1}\left(u_{1}^{\varepsilon}, \nabla u_{1}^{\varepsilon}\right) S^{\prime \prime}\left(u_{1}^{\varepsilon}\right) \nabla u_{1}^{\varepsilon} \\
& \quad+r_{1} \rho_{1}^{\varepsilon}\left(u_{1}^{\varepsilon}\right) S^{\prime}\left(u_{1}^{\varepsilon}\right)-r_{1} u_{2}^{\varepsilon} \mu_{1}\left(u_{1}^{\varepsilon}\right) S^{\prime}\left(u_{1}^{\varepsilon}\right)-\pi^{\varepsilon}\left(u_{1}^{\varepsilon}, u_{3}^{\varepsilon}\right) S^{\prime}\left(u_{1}^{\varepsilon}\right)+f^{\varepsilon} S^{\prime}\left(u_{1}^{\varepsilon}\right) .
\end{aligned}
$$

For any $S \in C^{\infty}(\mathbb{R})$ with supp $S^{\prime}$ compact and from the definition of $T_{k}\left(u_{1}^{\varepsilon}\right)$, we can rewrite (2.10) in the following way,

$$
\begin{aligned}
& \frac{\partial S\left(u_{1}^{\varepsilon}\right)}{\partial t}=\operatorname{div}\left(S^{\prime}\left(u_{1}^{\varepsilon}\right) \sigma_{1}\left(u_{2}^{\varepsilon}\right) d_{1}\left(T_{k}\left(u_{1}^{\varepsilon}\right), \nabla T_{k}\left(u_{1}^{\varepsilon}\right)\right)\right) \\
& \quad-\sigma_{1}\left(u_{2}^{\varepsilon}\right) d_{1}\left(T_{k}\left(u_{1}^{\varepsilon}\right), \nabla T_{k}\left(u_{1}^{\varepsilon}\right)\right) S^{\prime \prime}\left(u_{1}^{\varepsilon}\right) \nabla T_{k}\left(u_{1}^{\varepsilon}\right)+r_{1} \rho_{1}^{\varepsilon}\left(T_{k}\left(u_{1}^{\varepsilon}\right)\right) S^{\prime}\left(u_{1}^{\varepsilon}\right) \\
& -r_{1} u_{2}^{\varepsilon} \mu_{1}^{\varepsilon}\left(T_{k}\left(u_{1}^{\varepsilon}\right)\right) S^{\prime}\left(u_{1}^{\varepsilon}\right)-\pi^{\varepsilon}\left(T_{k}\left(u_{1}^{\varepsilon}\right), T_{k}\left(u_{3}^{\varepsilon}\right)\right) S^{\prime}\left(u_{1}^{\varepsilon}\right)+f^{\varepsilon} S^{\prime}\left(u_{1}^{\varepsilon}\right) .
\end{aligned}
$$

From $(2.11)$, we conclude that $\frac{\partial S\left(u_{1}^{\varepsilon}\right)}{\partial t}$ is bounded in $L^{1}\left(Q_{T}\right)+L^{2}\left(0, T ; H^{-1}(\Omega)\right)$. Similar arguments for $\frac{\partial S\left(u_{i}^{\varepsilon}\right)}{\partial t}, i=2,3$, proves the desired result. This completes the proof of the lemma.

Lemma 3. The solution triple $\left(u_{1}^{\varepsilon}, u_{2}^{\varepsilon}, u_{3}^{\varepsilon}\right)$ of the approximation system (2.1) is non-negative.

Proof. For $i=1,2,3$, consider $u_{i}^{-\varepsilon}=\sup \left(-u_{i}^{\varepsilon}, 0\right)$ and multiply (2.1) by $-T_{k}\left(u_{i}^{-\varepsilon}\right)$ respectively and integrating over $\Omega$, we get

$$
\begin{aligned}
& \frac{\mathrm{d}}{\mathrm{d} t} \int_{\Omega} \tilde{T}_{k}\left(u_{1}^{-\varepsilon}\right) \mathrm{d} x+\gamma_{1} \int_{\Omega} \sigma_{1}\left(u_{2}^{-\varepsilon}\right)\left|\nabla T_{k}\left(u_{1}^{-\varepsilon}\right)\right|^{2} \mathrm{~d} x \leq r_{1} \int_{\Omega} \rho_{1}\left(u_{1}^{-\varepsilon}\right) T_{k}\left(u_{1}^{-\varepsilon}\right) \mathrm{d} x \\
& +r_{1} \int_{\Omega} u_{2}^{-\varepsilon} \mu_{1}^{\varepsilon}\left(u_{1}^{-\varepsilon}\right) T_{k}\left(u_{1}^{-\varepsilon}\right) \mathrm{d} x-\int_{\Omega} \pi^{\varepsilon}\left(u_{1}^{-\varepsilon}, u_{3}^{-\varepsilon}\right) T_{k}\left(u_{1}^{-\varepsilon}\right) \mathrm{d} x+\int_{\Omega} f^{\varepsilon} T_{k}\left(u_{1}^{-\varepsilon}\right) \mathrm{d} x, \\
& \frac{\mathrm{d}}{\mathrm{d} t} \int_{\Omega} \tilde{T}_{k}\left(u_{2}^{-\varepsilon}\right) \mathrm{d} x+\gamma_{2} \int_{\Omega} \sigma_{2}\left(u_{1}^{-\varepsilon}\right)\left|\nabla T_{k}\left(u_{2}^{-\varepsilon}\right)\right|^{2} \mathrm{~d} x
\end{aligned}
$$




$$
\begin{aligned}
& \quad \leq r_{2} \int_{\Omega} \rho_{2}^{\varepsilon}\left(u_{2}^{-\varepsilon}\right) T_{k}\left(u_{2}^{-\varepsilon}\right) \mathrm{d} x+r_{2} \int_{\Omega} u_{1}^{-\varepsilon} \mu_{2}^{\varepsilon}\left(u_{2}^{-\varepsilon}\right) T_{k}\left(u_{2}^{-\varepsilon}\right) \mathrm{d} x+\int_{\Omega} g^{\varepsilon} T_{k}\left(u_{2}^{-\varepsilon}\right) \mathrm{d} x \\
& \frac{\mathrm{d}}{\mathrm{d} t} \int_{\Omega} \tilde{T}_{k}\left(u_{3}^{-\varepsilon}\right) \mathrm{d} x+\gamma_{3} \sigma_{3} \int_{\Omega}\left|\nabla T_{k}\left(u_{3}^{-\varepsilon}\right)\right|^{2} \mathrm{~d} x \\
& \quad \leq r_{3} \int_{\Omega} \rho_{2}^{\varepsilon}\left(u_{2}^{-\varepsilon}\right) T_{k}\left(u_{3}^{-\varepsilon}\right) \mathrm{d} x-\alpha_{3} \int_{\Omega} u_{3}^{-\varepsilon} T_{k}\left(u_{3}^{-\varepsilon}\right) \mathrm{d} x+\int_{\Omega} h^{\varepsilon} T_{k}\left(u_{2}^{-\varepsilon}\right) \mathrm{d} x .
\end{aligned}
$$

From the boundedness of solutions $\left(u_{1}^{\varepsilon}, u_{2}^{\varepsilon}, u_{3}^{\varepsilon}\right)$, continuity of $\sigma_{1}\left(u_{i}^{\varepsilon}\right), i=1,2$ and the non-negativity of $f^{\varepsilon}, g^{\varepsilon}, h^{\varepsilon}, u_{i, 0}^{\varepsilon}, i=1,2,3$ together with Young's inequality, we get

$$
\frac{\mathrm{d}}{\mathrm{d} t} \int_{\Omega} \tilde{T}_{k}\left(u_{i}^{-\varepsilon}\right)(t) \mathrm{d} x \leq 0, i=1,2,3 .
$$

This proves the required result.

Definition 2. We define the Lipschitz continuous function in the following form

$$
\Theta_{n}(z)=T_{n+1}(z)-T_{n}(z)= \begin{cases}0 & \text { if }|z| \leq n \\ (|z|-n) \operatorname{sgn}(z) & \text { if } n \leq|z| \leq n+1 \\ \operatorname{sgn}(z) & \text { if }|z| \geq n+1\end{cases}
$$

Obviously the function $\Theta_{n}(z)$ satisfies $\left\|\Theta_{n}(z)\right\|_{L^{\infty}(\mathbb{R})} \leq 1$, for any $n \geq 1$ and $\Theta_{n}(z) \rightarrow 0$, for any $n \geq 1$ and also $\Theta_{n}(z) \rightarrow 0$, for any $z$ when $n \rightarrow \infty$.

Lemma 4. The Lipschitz continuous function $\Theta_{n}\left(u_{i}\right), i=1,2,3$, for some $n>0$ and $\varepsilon>0$ satisfies

$$
\begin{gathered}
\lim _{n \rightarrow \infty} \varlimsup_{\varepsilon \rightarrow 0} \int_{0}^{t} \int_{\left(n \leq\left|u_{i}^{\varepsilon}\right| \leq n+1\right)} d_{i}\left(u_{i}^{\varepsilon}, \nabla u_{i}^{\varepsilon}\right) \nabla u_{i}^{\varepsilon} \mathrm{d} x \mathrm{~d} s=0 \text { and } \\
\Theta_{n}\left(u_{i}\right) \rightarrow 0 \text { strongly in } L^{2}\left(0, T ; H_{0}^{1}(\Omega)\right) \text { as } n \rightarrow \infty \text { and for } i=1,2,3 .
\end{gathered}
$$

Proof. Treating $\Theta_{n}\left(u_{1}^{\varepsilon}\right)$ as a test function and multiplying the first equation of (2.1) by $\Theta_{n}\left(u_{1}^{\varepsilon}\right)$ and integrating over $Q \times(0, t)$, we have

$$
\begin{aligned}
\int_{\Omega} \tilde{\Theta}_{n}\left(u_{1}^{\varepsilon}\right) \mathrm{d} x-\int_{\Omega} \tilde{\Theta}_{n}\left(u_{1,0}^{\varepsilon}\right) \mathrm{d} x+\int_{Q_{t}} \sigma_{1}^{\varepsilon}\left(u_{2}^{\varepsilon}\right) d_{1}\left(u_{1}^{\varepsilon}, \nabla u_{1}^{\varepsilon}\right) \nabla \Theta_{n}\left(u_{1}^{\varepsilon}\right) \mathrm{d} x \mathrm{~d} s \\
=r_{1} \int_{Q_{t}} \rho_{1}^{\varepsilon}\left(u_{1}^{\varepsilon}\right) \Theta_{n}\left(u_{1}^{\varepsilon}\right) \mathrm{d} x \mathrm{~d} s-r_{1} \int_{Q_{t}} u_{2}^{\varepsilon} \mu_{2}^{\varepsilon}\left(u_{1}^{\varepsilon}\right) \Theta_{n}\left(u_{1}^{\varepsilon}\right) \mathrm{d} x \mathrm{~d} s \\
\quad-\int_{Q_{t}} \pi^{\varepsilon}\left(u_{1}^{\varepsilon}, u_{3}^{\varepsilon}\right) \Theta_{n}\left(u_{1}^{\varepsilon}\right) \mathrm{d} x \mathrm{~d} s+\int_{Q_{t}} f^{\varepsilon} \Theta_{n}\left(u_{1}^{\varepsilon}\right) \mathrm{d} x \mathrm{~d} s
\end{aligned}
$$

for almost $t$ in $(0, T)$. Since $\Theta_{n}\left(u_{1}^{\varepsilon}\right) \geq 0$ for all $x \in \Omega$, we obtain

$$
\begin{aligned}
\int_{Q_{t}} & \sigma_{1}\left(u_{2}^{\varepsilon}\right) d_{1}\left(u_{1}^{\varepsilon}, \nabla u_{1}^{\varepsilon}\right) \nabla \Theta_{n}\left(u_{1}^{\varepsilon}\right) \mathrm{d} x \mathrm{~d} s \leq r_{1} \int_{Q_{t}} \rho_{1}^{\varepsilon}\left(u_{1}^{\varepsilon}\right) \Theta_{n}\left(u_{1}^{\varepsilon}\right) \mathrm{d} x \mathrm{~d} s \\
& -r_{1} \int_{Q_{t}} u_{2}^{\varepsilon} \mu_{2}^{\varepsilon}\left(u_{1}^{\varepsilon}\right) \Theta_{n}\left(u_{1}^{\varepsilon}\right) \mathrm{d} x \mathrm{~d} s-\int_{Q_{t}} \pi^{\varepsilon}\left(u_{1}^{\varepsilon}, u_{3}^{\varepsilon}\right) \Theta_{n}\left(u_{1}^{\varepsilon}\right) \mathrm{d} x \mathrm{~d} s \\
& +\int_{Q_{t}} f^{\varepsilon} \Theta_{n}\left(u_{1}^{\varepsilon}\right) \mathrm{d} x \mathrm{~d} s+\int_{\Omega} \tilde{\Theta}_{n}\left(u_{1,0}^{\varepsilon}(x)\right) \mathrm{d} x
\end{aligned}
$$


for all $t$ in $(0, T)$ and $\varepsilon<\frac{1}{n+1}$. For any subsequences $u_{i}^{\varepsilon}$ (still denoted by $u_{i}^{\varepsilon}$ ), Lemma 2 and (2.12) confirm that,

$$
\left.\begin{array}{ll}
u_{i}^{\varepsilon} \rightarrow u_{i} & \text { a.e. in } Q_{T}, i=1,2,3 \\
T_{k}\left(u_{i}^{\varepsilon}\right) \rightarrow T_{k}\left(u_{i}\right) & \text { weakly in } L^{2}\left(0, T ; H_{0}^{1}(\Omega)\right), i=1,2,3, \\
\Theta_{n}\left(u_{i}^{\varepsilon}\right) \rightarrow \Theta_{n}\left(u_{i}\right) & \text { weakly in } L^{2}\left(0, T ; H_{0}^{1}(\Omega)\right), i=1,2,3
\end{array}\right\}
$$

as $\varepsilon \rightarrow 0$ for any $k>0$ and $n \geq 1$. Using (H2), we can show that for $i=1,2,3$ $d_{i}\left(T_{k}\left(u_{i}^{\varepsilon}\right), \nabla T_{k}\left(u_{i}^{\varepsilon}\right)\right)$ is bounded in $L^{2}\left(Q_{T}\right)$. Therefore, for $i=1,2,3$,

$$
d_{i}\left(T_{k}\left(u_{i}^{\varepsilon}\right), \nabla T_{k}\left(u_{i}^{\varepsilon}\right)\right) \rightarrow \eta_{i, k} \text { weakly in } L^{2}\left(Q_{T}\right) \text { as } \varepsilon \rightarrow 0
$$

for $\eta_{i, k} \in L^{2}\left(Q_{T}\right)$. From (2.5)-(2.8) and Lemma 3, we have

$$
\begin{aligned}
& \sum_{i=1}^{3} \int_{\Omega} \tilde{T}_{k}\left(u_{i}^{\varepsilon}\right)(t) \mathrm{d} x \leq \int_{Q_{t}}\left(r_{1} \rho_{1}^{\varepsilon}\left(u_{1}^{\varepsilon}\right)-r_{1} u_{2}^{\varepsilon} \mu_{1}^{\varepsilon}\left(u_{1}^{\varepsilon}\right)-\pi^{\varepsilon}\left(u_{1}^{\varepsilon}, u_{3}^{\varepsilon}\right)+f^{\varepsilon}\right) T_{k}\left(u_{1}^{\varepsilon}\right) \mathrm{d} x \mathrm{~d} s \\
& \quad+\int_{Q_{t}}\left(r_{2} \rho_{2}^{\varepsilon}\left(u_{2}^{\varepsilon}\right)-u_{1}^{\varepsilon} \mu_{2}^{\varepsilon}\left(u_{2}^{\varepsilon}\right)+g^{\varepsilon}\right) T_{k}\left(u_{2}^{\varepsilon}\right) \mathrm{d} x \mathrm{~d} s \\
& \quad+\int_{Q_{t}}\left(r_{3} \rho_{2}^{\varepsilon}\left(u_{2}^{\varepsilon}\right)-\alpha_{3} u_{3}^{\varepsilon}+h^{\varepsilon}\right) T_{k}\left(u_{3}^{\varepsilon}\right) \mathrm{d} x \mathrm{~d} s+\sum_{i=1}^{3} \int_{\Omega} \tilde{T}_{k}\left(u_{i, 0}^{\varepsilon}\right)(x) \mathrm{d} x
\end{aligned}
$$

From the boundedness of the solution triple $\left(u_{1}^{\varepsilon}, u_{2}^{\varepsilon}, u_{3}^{\varepsilon}\right)$, Lemma 2 and use Young's inequality, we get

$$
\begin{aligned}
& \sum_{i=1}^{3} \int_{\Omega} \tilde{T}_{k}\left(u_{i}^{\varepsilon}\right) \mathrm{d} x \leq C_{k}+k\left(\left\|f^{\varepsilon}\right\|_{L^{2}\left(Q_{T}\right)}+\left\|g^{\varepsilon}\right\|_{L^{2}\left(Q_{T}\right)}\right. \\
& \left.\quad+\left\|h^{\varepsilon}\right\|_{L^{2}\left(Q_{T}\right)}+\sum_{i=1}^{3}\left\|u_{i, 0}^{\varepsilon}\right\|_{L^{2}(\Omega)}\right),
\end{aligned}
$$

where $C_{k}$ is a constant independent of $\varepsilon$. Taking lim inf as $\varepsilon$ tends to 0 in the above estimate and using the results (2.13) and Lemma 2, we have

$\sum_{i=1}^{3} \int_{\Omega} \tilde{T}_{k}\left(u_{i}\right) \mathrm{d} x \leq C_{k}+k\left(\|f\|_{L^{1}\left(Q_{T}\right)}+\|g\|_{L^{1}\left(Q_{T}\right)}+\|h\|_{L^{1}\left(Q_{T}\right)}+\sum_{i=1}^{3}\left\|u_{i, 0}\right\|_{L^{1}(\Omega)}\right)$.

From the definition of $\tilde{T}_{k}\left(u_{i}\right), i=1,2,3$, we deduce that

$$
\begin{aligned}
& \sum_{i=1}^{3} k \int_{\Omega}\left|u_{i}(x, t)\right| \mathrm{d} x \leq C_{k}+\frac{k^{2}}{2} \operatorname{meas}(\Omega) \\
& \quad+k\left(\|f\|_{L^{1}\left(Q_{T}\right)}+\|g\|_{L^{1}\left(Q_{T}\right)}+\|h\|_{L^{1}\left(Q_{T}\right)}+\sum_{i=1}^{3}\left\|u_{i, 0}^{\varepsilon}\right\|_{L^{1}(\Omega)}\right)
\end{aligned}
$$

for almost all $t \in(0, T)$ and $(2.15)$ shows that $u \in L^{\infty}\left(0, T ; L^{1}(\Omega)\right)$. 
Now (2.12) along with (2.13), proves that

$$
\begin{aligned}
& \varlimsup_{\varepsilon \rightarrow 0} \int_{Q_{t}} \sigma_{1}\left(u_{2}^{\varepsilon}\right) d_{1}\left(u_{1}^{\varepsilon}, \nabla u_{1}^{\varepsilon}\right) \nabla \Theta_{n}\left(u_{1}^{\varepsilon}\right) \mathrm{d} x \mathrm{~d} s \leq r_{1} \int_{Q_{t}} \rho_{1}\left(u_{1}\right) \Theta_{n}\left(u_{1}\right) \mathrm{d} x \mathrm{~d} s \\
& \quad-r_{1} \int_{Q_{t}} u_{2} \mu_{2}\left(u_{1}\right) \Theta_{n}\left(u_{1}\right) \mathrm{d} x \mathrm{~d} s-\int_{Q_{t}} \pi\left(u_{1}, u_{3}\right) \Theta_{n}\left(u_{1}\right) \mathrm{d} x \mathrm{~d} s \\
& \quad+\int_{Q_{t}} f \Theta_{n}\left(u_{1}\right) \mathrm{d} x \mathrm{~d} s+\int_{Q_{t}} \tilde{\Theta}_{n}\left(u_{1,0}(x)\right) \mathrm{d} x .
\end{aligned}
$$

Using $(H 1), \nabla \Theta_{n}\left(u^{\varepsilon}\right)=\chi_{\left(n \leq\left|u^{\varepsilon}\right| \leq n+1\right)} \nabla u^{\varepsilon}$, the continuity of $\sigma_{1}\left(u_{2}^{\varepsilon}\right)$ and the convergence results in (2.13), we have

$$
\begin{gathered}
\gamma_{1} \int_{Q_{t}}\left|\nabla \Theta_{n}\left(u_{1}\right)\right|^{2} \mathrm{~d} x \mathrm{~d} s \leq r_{1} \int_{Q_{t}} \rho_{1}\left(u_{1}\right) \Theta_{n}\left(u_{1}\right) \mathrm{d} x \mathrm{~d} s-r_{1} \int_{Q_{t}} u_{2} \mu_{2}\left(u_{1}\right) \Theta_{n}\left(u_{1}\right) \mathrm{d} x \mathrm{~d} s \\
-\int_{Q_{t}} \pi\left(u_{1}, u_{3}\right) \Theta_{n}\left(u_{1}\right) \mathrm{d} x \mathrm{~d} s+\int_{Q_{t}} f \Theta_{n}\left(u_{1}\right) \mathrm{d} x \mathrm{~d} s+\int_{Q_{t}} \tilde{\Theta}_{n}\left(u_{1,0}(x)\right) \mathrm{d} x .
\end{gathered}
$$

Since $\Theta_{n}\left(u_{1}\right) \rightarrow 0$ as $n \rightarrow \infty$, we have $\Theta_{n}\left(u_{1}\right) \rightarrow 0$ weakly in $L^{2}\left(0, T ; H_{0}^{1}(\Omega)\right)$. This leads to

$$
\begin{array}{ll}
\int_{Q_{t}} r_{1} \rho_{1}\left(u_{1}\right) \Theta_{n}\left(u_{1}\right) \mathrm{d} x \mathrm{~d} s \rightarrow 0, & \int_{Q_{t}} r_{1} u_{2} \mu_{2}\left(u_{1}\right) \Theta_{n}\left(u_{1}\right) \mathrm{d} x \mathrm{~d} s \rightarrow 0, \\
\int_{Q_{t}} \pi\left(u_{1}, u_{3}\right) \Theta_{n}\left(u_{1}\right) \mathrm{d} x \mathrm{~d} s \rightarrow 0, & \int_{Q_{t}} f \Theta_{n}\left(u_{1}\right) \mathrm{d} x \mathrm{~d} s \rightarrow 0,
\end{array}
$$

as $n \rightarrow \infty$. Also, $\tilde{\Theta}_{n}\left(u_{1,0}\right) \rightarrow 0$ a.e. in $\Omega$ as $n \rightarrow \infty$ and $\left\|\Theta_{n}\left(u_{1,0}\right)\right\| \leq$ $\left\|u_{1,0}\right\|_{L^{1}(\Omega)}$ implies $\int_{\Omega} \tilde{\Theta}_{n}\left(u_{1,0}\right) \mathrm{d} x$ tends to 0 as $n \rightarrow \infty$, which follows from the Lebesgue convergence theorem. Hence passing the lim-inf in (2.16) and (2.17), we get

$$
\begin{aligned}
& \lim _{n \rightarrow \infty} \varlimsup_{\varepsilon \rightarrow 0} \int_{0}^{t} \int_{\left(n \leq\left|u_{1}^{\varepsilon}\right| \leq n+1\right)} d_{1}\left(u_{1}^{\varepsilon}, \nabla u_{1}^{\varepsilon}\right) \nabla u_{1}^{\varepsilon} \mathrm{d} x \mathrm{~d} s=0, \\
& \Theta_{n}\left(u_{1}\right) \rightarrow 0 \text { strongly in } L^{2}\left(0, T ; H_{0}^{1}(\Omega)\right) \text { as } n \rightarrow \infty .
\end{aligned}
$$

Similarly, we can show that, for $i=1,2,3$

$$
\begin{aligned}
& \lim _{n \rightarrow \infty} \varlimsup_{\varepsilon \rightarrow 0} \int_{0}^{t} \int_{\left(n \leq\left|u_{i}^{\varepsilon}\right| \leq n+1\right)} d_{i}\left(u_{i}^{\varepsilon}, \nabla u_{i}^{\varepsilon}\right) \nabla u_{i}^{\varepsilon} \mathrm{d} x \mathrm{~d} s=0, \\
& \Theta_{n}\left(u_{i}\right) \rightarrow 0 \text { strongly in } L^{2}\left(0, T ; H_{0}^{1}(\Omega)\right) \text { as } n \rightarrow \infty .
\end{aligned}
$$

Definition 3. For $\mathrm{i}=1,2,3$, the time regularization for the function $T_{k}\left(u_{i}\right)$ is defined as

$$
\left(T_{k}\left(u_{i}\right)\right)_{\delta}=\delta \int_{-\infty}^{t} e^{\delta(s-t)} T_{k}\left(\overline{u_{i}(x, s)}\right) \mathrm{d} s, \text { where } \overline{u_{i}(x, s)}=\left\{\begin{array}{l}
u_{i}(x, s), \text { if } s>0 \\
u_{i, 0}(x), \text { if } s<0
\end{array}\right.
$$


Let us consider the unique solution $\left(T_{k}\left(u_{i}\right)\right)_{\delta} \in L^{\infty}\left(Q_{T}\right) \cap L^{2}\left(0, T ; H_{0}^{1}(\Omega)\right)$ of the monotone problem

$$
\left\{\begin{array}{l}
\frac{\partial}{\partial t}\left(T_{k}\left(u_{i}\right)\right)_{\delta}+\delta\left(\left(T_{k}\left(u_{i}\right)\right)_{\delta}-T_{k}\left(u_{i}\right)\right)=0 \text { in } Q_{T} \\
\left(T_{k}\left(u_{i}(x, 0)\right)\right)_{\delta}=T_{k}\left(u_{i, 0}(x)\right) \text { in } \Omega
\end{array}\right.
$$

for $\delta>0$ and $k>0$. From (2.18) and Lemma 2, we have $\frac{\partial}{\partial t}\left(T_{k}\left(u_{i}\right)\right)_{\delta} \in$ $L^{2}\left(0, T ; H_{0}^{1}(\Omega)\right)$.

Remark 1. For $i=1,2,3$, we have $\left(T_{k}\left(u_{i}\right)\right)_{\delta} \rightarrow T_{k}\left(u_{i}\right)$ a.e. in $Q_{T}$, weak-* in $L^{\infty}\left(Q_{T}\right)$ and strongly in $L^{2}\left(0, T ; H_{0}^{1}(\Omega)\right)$ as $\delta \rightarrow \infty$ and also

$$
\left\|\left(T_{k}\left(u_{i}\right)\right)_{\delta}\right\|_{L^{\infty}\left(Q_{T}\right)} \leq \max \left(\left\|T_{k}\left(u_{i}\right)\right\|_{L^{\infty}\left(Q_{T}\right)},\left\|T_{k}\left(u_{i, 0}\right)\right\|_{L^{\infty}(\Omega)}\right) \leq k,
$$

for any $\delta>0$ and $k \geq 0$.

Lemma 5. Let $k \geq 0$ be fixed and $S$ be an increasing $C^{\infty}(\mathbb{R})$ function such that $S(z)=z$ for $|z| \leq k$ and supp $S^{\prime}$ be compact. Then

$$
\lim _{n \rightarrow \infty} \varlimsup_{\varepsilon \rightarrow 0} \int_{0}^{T} \int_{Q_{t}} \frac{\partial S\left(u_{i}^{\varepsilon}\right)}{\partial t}\left(T_{k}\left(u_{i}^{\varepsilon}\right)-\left(T_{k}\left(u_{i}\right)\right)_{\delta}\right) \mathrm{d} x \mathrm{~d} s \mathrm{~d} t \geq 0 .
$$

Proof. The proof is as similar as of Lemma 1 in [9].

Lemma 6. For $i=1,2,3$, and $\eta_{i, k}$ which is defined in (2.14), the subsequence of $u_{i}^{\varepsilon}$ (still denoted by $u_{i}^{\varepsilon}$ ) satisfies, for $i=1,2,3$,

$$
\varlimsup_{\varepsilon \rightarrow 0} \int_{0}^{T} \int_{Q_{t}} d_{i}\left(T_{k}\left(u_{i}^{\varepsilon}\right), \nabla T_{k}\left(u_{i}^{\varepsilon}\right)\right) \nabla T_{k}\left(u_{i}^{\varepsilon}\right) \mathrm{d} x \mathrm{~d} s \mathrm{~d} t \leq \int_{0}^{T} \int_{Q_{t}} \eta_{i, k} \nabla T_{k}\left(u_{i}\right) \mathrm{d} x \mathrm{~d} s \mathrm{~d} t .
$$

Proof. Let us introduce $S_{n}$ to be a sequence of increasing $C^{\infty}(\mathbb{R})$ functions such that

$$
\begin{aligned}
& S_{n}(z)=z, \text { for }|z| \leq n, \\
& \operatorname{supp} S_{n}^{\prime} \subset[-(n+1),(n+1)], \quad\left\|S_{n}^{\prime \prime}\right\|_{L^{\infty}(\mathbb{R})} \leq 1 .
\end{aligned}
$$

Multiply the first equation of $(2.1)$ by $S_{n}^{\prime}\left(u_{1}^{\varepsilon}\right)$ to get

$$
\begin{aligned}
& \frac{\partial S_{n}\left(u_{1}^{\varepsilon}\right)}{\partial t}=\operatorname{div}\left(S_{n}^{\prime}\left(u_{1}^{\varepsilon}\right) \sigma_{1}\left(u_{2}^{\varepsilon}\right) d_{1}\left(u_{1}^{\varepsilon}, \nabla u_{1}^{\varepsilon}\right)\right)-\sigma_{1}\left(u_{2}^{\varepsilon}\right) d_{1}\left(u_{1}^{\varepsilon}, \nabla u_{1}^{\varepsilon}\right) S_{n}^{\prime \prime}\left(u_{1}^{\varepsilon}\right) \nabla u_{1}^{\varepsilon} \\
& +r_{1} \rho_{1}^{\varepsilon}\left(u_{1}^{\varepsilon}\right) S_{n}^{\prime}\left(u_{1}^{\varepsilon}\right)-r_{1} u_{2}^{\varepsilon} \mu_{1}\left(u_{1}^{\varepsilon}\right) S_{n}^{\prime}\left(u_{1}^{\varepsilon}\right)-\pi^{\varepsilon}\left(u_{1}^{\varepsilon}, u_{3}^{\varepsilon}\right) S_{n}^{\prime}\left(u_{1}^{\varepsilon}\right)+f^{\varepsilon} S_{n}^{\prime}\left(u_{1}^{\varepsilon}\right) .
\end{aligned}
$$

By similar procedure, we obtain that, for $i=1,2,3$

$$
\frac{\partial}{\partial t} S_{n}\left(u_{i}^{\varepsilon}\right) \in L^{1}\left(Q_{T}\right)+L^{2}\left(0, T ; H^{-1}(\Omega)\right) .
$$

For fixed $k>0, \delta>0$ and $\varepsilon>0$, set

$$
\Psi_{\delta}^{\varepsilon}=T_{k}\left(u_{i}^{\varepsilon}\right)-\left(T_{k}\left(u_{i}\right)\right)_{\delta}, \quad i=1,2,3 .
$$


Multiplying (2.20) by $\Psi_{\delta}^{\varepsilon}$ and integrating over $Q_{t} \times(0, T)$, we get

$$
\begin{aligned}
\int_{Q} \frac{\partial S_{n}\left(u_{1}^{\varepsilon}\right)}{\partial t} \Psi_{\delta}^{\varepsilon} \mathrm{d} x \mathrm{~d} s \mathrm{~d} t=-\int_{Q} S_{n}^{\prime}\left(u_{1}^{\varepsilon}\right) \sigma_{1}\left(u_{2}^{\varepsilon}\right) d_{1}\left(u_{1}^{\varepsilon}, \nabla u_{1}^{\varepsilon}\right) \nabla \Psi_{\delta}^{\varepsilon} \mathrm{d} x \mathrm{~d} s \mathrm{~d} t \\
\quad-\int_{Q} S_{n}^{\prime \prime}\left(u_{1}^{\varepsilon}\right) \sigma_{1}\left(u_{2}^{\varepsilon}\right) d_{1}\left(u_{1}^{\varepsilon}, \nabla u_{1}^{\varepsilon}\right) \nabla u_{1}^{\varepsilon} \Psi_{\delta}^{\varepsilon} \mathrm{d} x \mathrm{~d} s \mathrm{~d} t \\
\quad+r_{1} \int_{Q} S_{n}^{\prime}\left(u_{1}^{\varepsilon}\right) \rho_{1}^{\varepsilon}\left(u_{1}^{\varepsilon}\right) \Psi_{\delta}^{\varepsilon} \mathrm{d} x \mathrm{~d} s \mathrm{~d} t-r_{1} \int_{Q} S_{n}^{\prime}\left(u_{1}^{\varepsilon}\right) u_{2}^{\varepsilon} \mu_{1}^{\varepsilon}\left(u_{1}^{\varepsilon}\right) \Psi_{\delta}^{\varepsilon} \mathrm{d} x \mathrm{~d} s \mathrm{~d} t \\
\quad-\int_{Q} S_{n}^{\prime}\left(u_{1}^{\varepsilon}\right) \pi^{\varepsilon}\left(u_{1}^{\varepsilon}, u_{3}^{\varepsilon}\right) \Psi_{\delta}^{\varepsilon} \mathrm{d} x \mathrm{~d} s \mathrm{~d} t+\int_{Q} f^{\varepsilon} S_{n}^{\prime}\left(u_{1}^{\varepsilon}\right) \Psi_{\delta}^{\varepsilon} \mathrm{d} x \mathrm{~d} s \mathrm{~d} t
\end{aligned}
$$

where $Q=Q_{t} \times(0, T)$. From (2.21) for fixed $\delta>0, \Psi_{\delta}^{\varepsilon} \rightarrow T_{k}\left(u_{i}\right)-\left(T_{k}\left(u_{i}\right)\right)_{\delta}, i=$ $1,2,3$ weakly in $L^{2}\left(0, T ; H_{0}^{1}(\Omega)\right)$ as $\varepsilon \rightarrow 0$. By Remark 1 , we conclude that $\left\|\Psi_{\delta}^{\varepsilon}\right\|_{L^{\infty}(\Omega)} \leq 2 k$, for any $\varepsilon>0$ and $\delta>0$. Therefore, the boundedness of $\Psi_{\delta}^{\varepsilon}$ shows that for fixed $\delta>0, \Psi_{\delta}^{\varepsilon} \rightarrow T_{k}\left(u_{i}\right)-\left(T_{k}\left(u_{i}\right)\right)_{\delta}, i=1,2,3$ a.e. in $Q_{T}$ and $L^{\infty}\left(Q_{T}\right)$ weak-* as $\varepsilon \rightarrow 0$.

By the definition of $S_{n}$, we have $\operatorname{supp} S_{n}^{\prime \prime} \subset[-(n+1),(n+1)] \cup[n, n+1]$ for any $n \geq 1$. As a consequence

$$
\begin{aligned}
& \left|\int_{Q} S_{n}^{\prime \prime}\left(u_{1}^{\varepsilon}\right) \sigma_{1}\left(u_{2}^{\varepsilon}\right) d_{1}\left(u_{1}^{\varepsilon}, \nabla u_{1}^{\varepsilon}\right) \nabla u_{1}^{\varepsilon} \Psi_{\delta}^{\varepsilon} \mathrm{d} x \mathrm{~d} s \mathrm{~d} t\right| \leq T\left\|S_{n}^{\prime \prime}\left(u_{1}^{\varepsilon}\right)\right\|_{L^{\infty}((R))} \\
& \quad \times\left\|\sigma_{1}\left(u_{2}^{\varepsilon}\right)\right\|_{L^{\infty}\left(Q_{T}\right)}\left\|\Psi_{\delta}^{\varepsilon}\right\|_{L^{\infty}\left(Q_{T}\right)} \int_{\left((x, t) ; n \leq\left|u_{1}^{\varepsilon}\right| \leq n+1\right)} d_{1}\left(u_{1}^{\varepsilon}, \nabla u_{1}^{\varepsilon}\right) \nabla u_{1}^{\varepsilon} \mathrm{d} x \mathrm{~d} s
\end{aligned}
$$

for any $n \geq 1, \varepsilon \leq \frac{1}{n+1}$ and $\delta>0$. From $\left\|\Psi_{\delta}^{\varepsilon}\right\|_{L^{\infty}\left(Q_{T}\right)} \leq 2 k$ and (2.19), it can be easily obtained that

$$
\begin{aligned}
& \varlimsup_{\delta \rightarrow \infty} \varlimsup_{\varepsilon \rightarrow 0}\left|\int_{Q} S_{n}^{\prime \prime}\left(u_{1}^{\varepsilon}\right) \sigma_{1}\left(u_{2}^{\varepsilon}\right) d_{1}\left(u_{1}^{\varepsilon}, \nabla u_{1}^{\varepsilon}\right) \nabla u_{1}^{\varepsilon} \Psi_{\delta}^{\varepsilon} \mathrm{d} x \mathrm{~d} s \mathrm{~d} t\right| \\
& \leq C \varlimsup_{\varepsilon \rightarrow 0} \int_{\left((x, t) ; n \leq\left|u_{1}^{\varepsilon}\right| \leq n+1\right)} d_{1}\left(u_{1}^{\varepsilon}, \nabla u_{1}^{\varepsilon}\right) \nabla u_{1}^{\varepsilon} \mathrm{d} x \mathrm{~d} s,
\end{aligned}
$$

for any $n \geq 1$, where the constant $C$ depends only on $T$ and $k$. Hence, by Lemma 4, we achieve that

$$
\lim _{n \rightarrow \infty} \varlimsup_{\delta \rightarrow \infty} \varlimsup_{\varepsilon \rightarrow 0}\left|\int_{Q} S_{n}^{\prime \prime}\left(u_{1}^{\varepsilon}\right) \sigma_{1}\left(u_{2}^{\varepsilon}\right) d_{1}\left(u_{1}^{\varepsilon}, \nabla u_{1}^{\varepsilon}\right) \nabla u_{1}^{\varepsilon} \Psi_{\delta}^{\varepsilon} \mathrm{d} x \mathrm{~d} s \mathrm{~d} t\right|=0 .
$$

For some $n \geq 1$, we have

$$
r_{1} S_{n}^{\prime}\left(u_{1}^{\varepsilon}\right) \rho_{1}^{\varepsilon}\left(u_{1}^{\varepsilon}\right) \Psi_{\delta}^{\varepsilon}=r_{1} S_{n}^{\prime}\left(u_{1}^{\varepsilon}\right) \rho_{1}^{\varepsilon}\left(T_{n+1}\left(u_{1}^{\varepsilon}\right)\right) \Psi_{\delta}^{\varepsilon}
$$

a.e. in $Q_{T}$, since supp $S_{n}^{\prime} \subset[-(n+1),(n+1)]$. Definition of $\rho_{1}^{\varepsilon}\left(u_{1}^{\varepsilon}\right)$ and the result $(2.13)$ lead to $S_{n}^{\prime}\left(u^{\varepsilon}\right) \rho_{1}^{\varepsilon}\left(T_{n+1}\left(u_{1}^{\varepsilon}\right)\right) \rightarrow S_{n}^{\prime}(u) \rho_{1}\left(T_{n+1}\left(u_{1}\right)\right)$ a.e. in $Q_{T}$ and in $L^{\infty}\left(Q_{T}\right)$ weak-* as $\varepsilon \rightarrow 0$. This proves

$\lim _{\varepsilon \rightarrow 0} \int_{Q} r_{1} S_{n}^{\prime}\left(u_{1}^{\varepsilon}\right) \rho_{1}^{\varepsilon}\left(u_{1}^{\varepsilon}\right) \Psi_{\delta}^{\varepsilon} \mathrm{d} x \mathrm{~d} s \mathrm{~d} t=\int_{Q} r_{1} S_{n}^{\prime}\left(u_{1}\right) \rho_{1}\left(u_{1}\right)\left(T_{k}\left(u_{1}\right)-\left(T_{k}\left(u_{1}\right)\right)_{\delta}\right) \mathrm{d} x \mathrm{~d} s \mathrm{~d} t$, 
for any $\delta>0$. Using Remark 1 , we set

$$
\lim _{\delta \rightarrow \infty} \lim _{\varepsilon \rightarrow 0} \int_{Q} r_{1} S_{n}^{\prime}\left(u_{1}^{\varepsilon}\right) \rho_{1}^{\varepsilon}\left(u_{1}^{\varepsilon}\right) \Psi_{\delta}^{\varepsilon} \mathrm{d} x \mathrm{~d} s \mathrm{~d} t=0 .
$$

Similarly, we can show that

$$
\begin{aligned}
& \lim _{\delta \rightarrow \infty} \lim _{\varepsilon \rightarrow 0} \int_{Q} r_{1} S_{n}^{\prime}\left(u_{1}^{\varepsilon}\right) u_{2}^{\varepsilon} \mu_{1}\left(u_{1}^{\varepsilon}\right) \Psi_{\delta}^{\varepsilon} \mathrm{d} x \mathrm{~d} s \mathrm{~d} t=0, \\
& \lim _{\delta \rightarrow \infty} \lim _{\varepsilon \rightarrow 0} \int_{Q} S_{n}^{\prime}\left(u_{1}^{\varepsilon}\right) \pi\left(u_{1}^{\varepsilon}, u_{3}^{\varepsilon}\right) \Psi_{\delta}^{\varepsilon} \mathrm{d} x \mathrm{~d} s \mathrm{~d} t=0 .
\end{aligned}
$$

Since $f S_{n}^{\prime}\left(u_{1}\right) \in L^{1}\left(Q_{T}\right),(2.13)$ and Remark 1 lead to,

$$
\lim _{\delta \rightarrow \infty} \lim _{\varepsilon \rightarrow 0} \int_{Q} f^{\varepsilon} S_{n}^{\prime}\left(u_{1}^{\varepsilon}\right) \Psi_{\delta}^{\varepsilon} \mathrm{d} x \mathrm{~d} s \mathrm{~d} t=0 .
$$

Consequently from Lemma 5 and the definition of $\Psi_{\delta}^{\varepsilon}$, we have

$$
\lim _{\delta \rightarrow \infty} \lim _{\varepsilon \rightarrow 0} \int_{Q} \frac{\partial S_{n}\left(u_{1}^{\varepsilon}\right)}{\partial t} \Psi_{\delta}^{\varepsilon} \mathrm{d} x \mathrm{~d} s \mathrm{~d} t \geq 0 \quad \text { for any } n \geq k .
$$

From (2.23)-(2.24) along with (2.22) and (2.25), we get

$$
\lim _{n \rightarrow \infty} \varlimsup_{\delta \rightarrow \infty} \varlimsup_{\varepsilon \rightarrow 0} \int_{Q} S_{n}^{\prime}\left(u_{1}^{\varepsilon}\right) d_{1}\left(u_{1}^{\varepsilon}, \nabla u_{1}^{\varepsilon}\right) \nabla \Psi_{\delta}^{\varepsilon} \mathrm{d} x \mathrm{~d} s \mathrm{~d} t \leq 0 .
$$

Using the definition of $S_{n}$ and (2.26) to get

$$
\begin{aligned}
& \varlimsup_{\varepsilon \rightarrow 0} \int_{Q} d_{1}\left(u_{1}^{\varepsilon}, \nabla u_{1}^{\varepsilon}\right) \nabla T_{k}\left(u_{1}^{\varepsilon}\right) \mathrm{d} x \mathrm{~d} s \mathrm{~d} t \\
& \quad \leq \lim _{n \rightarrow \infty} \varlimsup_{\delta \rightarrow \infty} \varlimsup_{\varepsilon \rightarrow 0} \int_{Q} S_{n}^{\prime}\left(u_{1}^{\varepsilon}\right) d_{1}\left(u_{1}^{\varepsilon}, \nabla u_{1}^{\varepsilon}\right) \nabla\left(T_{k}\left(u_{1}^{\varepsilon}\right)\right)_{\delta} \mathrm{d} x \mathrm{~d} s \mathrm{~d} t \quad \text { for } \quad k \leq n
\end{aligned}
$$

We know that

$$
S_{n}^{\prime}\left(u_{1}^{\varepsilon}\right) d_{1}\left(u_{1}^{\varepsilon}, \nabla u_{1}^{\varepsilon}\right)=S_{n}^{\prime}\left(u_{1}^{\varepsilon}\right) d_{1}\left(T_{n+1}\left(u_{1}^{\varepsilon}\right), \nabla T_{n+1}\left(u_{1}^{\varepsilon}\right)\right), \text { for } \varepsilon<\frac{1}{n+1} .
$$

From (2.14), we have $S_{n}^{\prime}\left(u_{1}^{\varepsilon}\right) d_{1}\left(T_{n+1}\left(u_{1}^{\varepsilon}\right), \nabla\left(T_{n+1}\left(u_{1}^{\varepsilon}\right)\right) \rightarrow S_{n}^{\prime}\left(u_{1}^{\varepsilon}\right) \eta_{1, n+1}\right.$ weakly in $L^{2}\left(Q_{T}\right)$ as $\varepsilon \rightarrow 0$. This helps us to prove that

$$
\begin{aligned}
\varlimsup_{\varepsilon \rightarrow 0} \int_{Q} d_{1}\left(T_{k}\left(u_{1}^{\varepsilon}\right),\right. & \left.\nabla T_{k}\left(u_{1}^{\varepsilon}\right)\right) \nabla T_{k}\left(u_{1}^{\varepsilon}\right) \mathrm{d} x \mathrm{~d} s \mathrm{~d} t \\
& \leq \lim _{n \rightarrow \infty} \int_{Q} S_{n}^{\prime}\left(u_{1}\right) \eta_{1, n+1} \nabla T_{k}\left(u_{1}\right) \mathrm{d} x \mathrm{~d} s \mathrm{~d} t \\
& =\int_{Q} \eta_{1, n+1} \nabla T_{k}\left(u_{1}\right) \mathrm{d} x \mathrm{~d} s \mathrm{~d} t \text { for any } n \leq 1 .
\end{aligned}
$$

For any $k \leq n$, we have

$d_{1}\left(T_{n+1}\left(u_{1}^{\varepsilon}\right), \nabla T_{n+1}\left(u_{1}^{\varepsilon}\right)\right)_{\chi\left\{\left|u_{1}^{\varepsilon}\right| \leq k\right\}}=d_{1}\left(T_{k}\left(u_{1}^{\varepsilon}\right), \nabla T_{k}\left(u_{1}^{\varepsilon}\right)\right)_{\chi\left\{\left|u^{\varepsilon}\right| \leq k\right\}}$ a.e. in $Q_{T}$. 
Above equation with (2.13) and (2.14), implies that

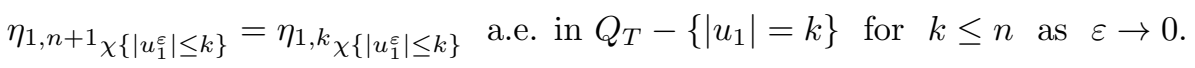

Therefore (2.27) becomes

$$
\varlimsup_{\varepsilon \rightarrow 0} \int_{Q} d_{1}\left(T_{k}\left(u_{1}^{\varepsilon}\right), \nabla T_{k}\left(u_{1}^{\varepsilon}\right)\right) \nabla T_{k}\left(u_{1}^{\varepsilon}\right) \mathrm{d} x \mathrm{~d} s \mathrm{~d} t=\int_{Q} \eta_{1, k} \nabla T_{k}\left(u_{1}\right) \mathrm{d} x \mathrm{~d} s \mathrm{~d} t .
$$

Similarly, we get

$$
\varlimsup_{\varepsilon \rightarrow 0} \int_{Q} d_{i}\left(T_{k}\left(u_{i}^{\varepsilon}\right), \nabla T_{k}\left(u_{i}^{\varepsilon}\right)\right) \nabla T_{k}\left(u_{i}^{\varepsilon}\right) \mathrm{d} x \mathrm{~d} s \mathrm{~d} t=\int_{Q} \eta_{i, k} \nabla T_{k}\left(u_{i}\right) \mathrm{d} x \mathrm{~d} s \mathrm{~d} t .
$$

Lemma 7. For any $k \geq 0$ and $i=1,2,3$, we have

$$
\begin{gathered}
\varlimsup_{\varepsilon \rightarrow 0} \int_{Q}\left[d_{i}\left(T_{k}\left(u_{i}^{\varepsilon}\right), \nabla T_{k}\left(u_{i}^{\varepsilon}\right)\right)-d_{i}\left(T_{k}\left(u_{i}\right), \nabla T_{k}\left(u_{i}\right)\right)\right] \\
\quad \times\left[\nabla T_{k}\left(u_{i}^{\varepsilon}\right)-\nabla T_{k}\left(u_{i}\right)\right] \mathrm{d} x \mathrm{~d} s \mathrm{~d} t=0 .
\end{gathered}
$$

Proof. From the monotone character (H3), for any $k \geq 0$, we have

$$
\begin{aligned}
& \varlimsup_{\varepsilon \rightarrow 0} \int_{Q}\left[d_{i}\left(T_{k}\left(u_{i}^{\varepsilon}\right), \nabla T_{k}\left(u_{i}^{\varepsilon}\right)\right)-d_{i}\left(T_{k}\left(u_{i}\right), \nabla T_{k}\left(u_{i}\right)\right)\right] \\
& \quad \times\left[\nabla T_{k}\left(u_{i}^{\varepsilon}\right)-\nabla T_{k}\left(u_{i}\right)\right] \mathrm{d} x \mathrm{~d} s \mathrm{~d} t \geq 0
\end{aligned}
$$

By $(H 2)$ one can remark that $\left|d_{i}\left(T_{k}\left(u_{i}\right), \nabla T_{k}\left(u_{i}\right)\right)\right| \leq C_{k}\left(1+\left|\nabla T_{k}\left(u_{i}\right)\right|\right)$ a.e. in $Q_{T}$. Then by Lemma $4,(2.13)$ and $(2.14)$, we have

$$
\begin{aligned}
\varlimsup_{\varepsilon \rightarrow 0} \int_{Q}\left[d _ { i } \left(T_{k}\left(u_{i}^{\varepsilon}\right)\right.\right. & \left.\left., \nabla T_{k}\left(u_{i}^{\varepsilon}\right)\right)-d_{i}\left(T_{k}\left(u_{i}\right), \nabla T_{k}\left(u_{i}\right)\right)\right] \\
\times & {\left[\nabla T_{k}\left(u_{i}^{\varepsilon}\right)-\nabla T_{k}\left(u_{i}\right)\right] \mathrm{d} x \mathrm{~d} s \mathrm{~d} t=0 \text { for any } k \geq 0 . }
\end{aligned}
$$

Lemma 8. For fixed $k \geq 0$ and $i=1,2,3$, we have $\eta_{i, k}=d_{i}\left(T_{k}\left(u_{i}\right), \nabla T_{k}\left(u_{i}\right)\right)$ a.e. in $Q_{T}$ and

$$
d_{i}\left(T_{k}\left(u_{i}^{\varepsilon}\right), \nabla T_{k}\left(u_{i}^{\varepsilon}\right)\right) \nabla T_{k}\left(u_{i}^{\varepsilon}\right) \rightarrow d_{i}\left(T_{k}\left(u_{i}\right), \nabla T_{k}\left(u_{i}\right)\right) \nabla T_{k}\left(u_{i}\right)
$$

weakly in $L^{1}\left(Q_{T}\right)$.

Proof. For any $k>0$ and $0<\varepsilon<\frac{1}{k}$, Lemma 6, (2.13) implies that, for $i=1,2,3$,

$$
\varlimsup_{\varepsilon \rightarrow 0} \int_{Q} d_{i}\left(T_{k}\left(u_{i}^{\varepsilon}\right), \nabla T_{k}\left(u_{i}^{\varepsilon}\right)\right) \nabla T_{k}\left(u_{i}^{\varepsilon}\right) \mathrm{d} x \mathrm{~d} s \mathrm{~d} t=\int_{Q} \eta_{i, k} \nabla T_{k}\left(u_{i}\right) \mathrm{d} x \mathrm{~d} s \mathrm{~d} t .
$$


Minty's type of arguments, in view of (2.13), shows that $d_{i}\left(T_{k}\left(u_{i}\right), \nabla T_{k}\left(u_{i}\right)\right)=$ $\eta_{i, k}$ for any $k \geq 0$. This proves the first result of Lemma.

For any $k \geq 0, T^{\prime}<T$, Lemma 7 shows that,

$$
\left[d_{i}\left(T_{k}\left(u_{i}^{\varepsilon}\right), \nabla T_{k}\left(u_{i}^{\varepsilon}\right)\right)-d_{i}\left(T_{k}\left(u_{i}\right), \nabla T_{k}\left(u_{i}\right)\right)\right]\left[\nabla T_{k}\left(u_{i}^{\varepsilon}\right)-\nabla T_{k}\left(u_{i}\right)\right] \rightarrow 0
$$

strongly in $L^{1}\left(\Omega \times\left(0, T^{\prime}\right)\right)$, as $\varepsilon \rightarrow 0$. By $(2.13)$, the first result of the present Lemma implies that

$$
\begin{aligned}
& d_{i}\left(T_{k}\left(u_{i}^{\varepsilon}\right), \nabla T_{k}\left(u_{i}\right)\right) \rightarrow d_{i}\left(T_{k}\left(u_{i}\right), \nabla T_{k}\left(u_{i}\right)\right) \text { weakly in } L^{1}\left(Q_{T}\right), \\
& d_{i}\left(T_{k}\left(u_{i}\right), \nabla T_{k}\left(u_{i}^{\varepsilon}\right)\right) \rightarrow d_{i}\left(T_{k}\left(u_{i}\right), \nabla T_{k}\left(u_{i}\right)\right) \text { weakly in } L^{1}\left(Q_{T}\right) .
\end{aligned}
$$

Hence $d_{i}\left(T_{k}\left(u_{i}^{\varepsilon}\right), \nabla T_{k}\left(u_{i}^{\varepsilon}\right)\right) \rightarrow d_{i}\left(T_{k}\left(u_{i}\right), \nabla T_{k}\left(u_{i}\right)\right)$ weakly in $L^{1}\left(\Omega \times\left(0, T^{\prime}\right)\right)$ for any $T^{\prime}<T$ as $\varepsilon \rightarrow 0$. According to the definition of $d_{i}(\eta, \zeta)$, the assumptions hold true for all time $T$. Hence $d_{i}\left(T_{k}\left(u_{i}^{\varepsilon}\right), \nabla T_{k}\left(u_{i}^{\varepsilon}\right)\right) \rightarrow d_{i}\left(T_{k}\left(u_{i}\right), \nabla T_{k}\left(u_{i}\right)\right)$ weakly in $L^{1}\left(Q_{T}\right)$ holds.

Lemma 9. For any $n \geq 0$ and $i=1,2,3$,

$$
\int_{\left\{(x, t) \in Q_{T} ; n \leq\left|u_{i}\right| \leq n+1\right\}} d_{i}\left(u_{i}, \nabla u_{i}\right) \nabla u_{i} \mathrm{~d} x \mathrm{~d} t \rightarrow 0 \quad \text { as } n \rightarrow \infty .
$$

Proof.

$$
\begin{aligned}
\lim _{\varepsilon \rightarrow 0} & \int_{\left\{(x, t) \in Q_{T} ; n \leq\left|u_{i}^{\varepsilon}\right| \leq n+1\right\}} d_{i}\left(u_{i}^{\varepsilon}, \nabla u_{i}^{\varepsilon}\right) \nabla u_{i}^{\varepsilon} \mathrm{d} x \mathrm{~d} t=\lim _{\varepsilon \rightarrow 0} \int_{Q_{T}} d_{i}\left(u_{i}^{\varepsilon}, \nabla u_{i}^{\varepsilon}\right) \nabla\left(T_{n+1}\left(u_{i}^{\varepsilon}\right)-T_{n}\left(u_{i}^{\varepsilon}\right)\right) \mathrm{d} x \mathrm{~d} t \\
= & \int_{Q_{T}} d_{i}\left(u_{i}, \nabla u_{i}\right) \nabla\left(T_{n+1}\left(u_{i}\right)\right) \mathrm{d} x \mathrm{~d} t-\int_{Q_{T}} d_{i}\left(u_{i}, \nabla u_{i}\right) \nabla\left(T_{n}\left(u_{i}\right)\right) \mathrm{d} x \mathrm{~d} t \\
= & \int_{\left\{(x, t) \in Q_{T} ; n \leq\left|u_{i}\right| \leq n+1\right\}} d_{1}\left(u_{i}, \nabla u_{i}\right) \nabla u_{i} \mathrm{~d} x \mathrm{~d} t \text { for any } n \geq 0 .
\end{aligned}
$$

Using Lemma 4 together with the above inequality, we get

$$
\int_{\left\{(x, t) \in Q_{T} ; n \leq\left|u_{i}\right| \leq n+1\right\}} d_{i}\left(u_{i}, \nabla u_{i}\right) \nabla u_{i} \mathrm{~d} x \mathrm{~d} t \rightarrow 0 \quad \text { as } n \rightarrow \infty .
$$

Now, we prove the main theorem of the work. We have used the Lemma 2 and Aubin type of lemma to prove the Theorem 1.

Proof. From the system (2.1), we have

$$
\begin{aligned}
& \frac{\partial S\left(u_{1}^{\varepsilon}\right)}{\partial t}-\operatorname{div}\left(S^{\prime}\left(u_{1}^{\varepsilon}\right) \sigma_{1}\left(u_{2}^{\varepsilon}\right) d_{1}\left(u_{1}^{\varepsilon}, \nabla u_{1}^{\varepsilon}\right)\right)+\sigma_{1}\left(u_{2}^{\varepsilon}\right) d_{1}\left(u_{1}^{\varepsilon}, \nabla u_{1}^{\varepsilon}\right) S^{\prime \prime}\left(u_{1}^{\varepsilon}\right) \nabla u_{1}^{\varepsilon} \\
& \quad=r_{1} \rho_{1}^{\varepsilon}\left(u_{1}^{\varepsilon}\right) S^{\prime}\left(u_{1}^{\varepsilon}\right)-r_{1} u_{2}^{\varepsilon} \mu_{1}^{\varepsilon}\left(u_{1}^{\varepsilon}\right) S^{\prime}\left(u_{1}^{\varepsilon}\right)-\pi^{\varepsilon}\left(u_{1}^{\varepsilon}, u_{3}^{\varepsilon}\right) S^{\prime}\left(u_{1}^{\varepsilon}\right)+f^{\varepsilon} S^{\prime}\left(u_{1}^{\varepsilon}\right), \\
& \frac{\partial S\left(u_{2}^{\varepsilon}\right)}{\partial t}-\operatorname{div}\left(S^{\prime}\left(u_{2}^{\varepsilon}\right) \sigma_{2}\left(u_{1}^{\varepsilon}\right) d_{2}\left(u_{2}^{\varepsilon}, \nabla u_{2}^{\varepsilon}\right)\right)+\sigma_{2}\left(u_{1}^{\varepsilon}\right) d_{2}\left(u_{2}^{\varepsilon}, \nabla u_{2}^{\varepsilon}\right) S^{\prime \prime}\left(u_{2}^{\varepsilon}\right) \nabla u_{2}^{\varepsilon} \\
& \quad=r_{2} \rho_{2}^{\varepsilon}\left(u_{2}^{\varepsilon}\right) S^{\prime}\left(u_{2}^{\varepsilon}\right)-r_{2} u_{1}^{\varepsilon} \mu_{2}^{\varepsilon}\left(u_{2}^{\varepsilon}\right) S^{\prime}\left(u_{2}^{\varepsilon}\right)+g^{\varepsilon} S^{\prime}\left(u_{2}^{\varepsilon}\right), \\
& \frac{\partial S\left(u_{3}^{\varepsilon}\right)}{\partial t}-\sigma_{3} \operatorname{div}\left(S^{\prime}\left(u_{3}^{\varepsilon}\right) d_{3}\left(u_{3}^{\varepsilon}, \nabla u_{3}^{\varepsilon}\right)\right)+\sigma_{3} d_{3}\left(u_{3}^{\varepsilon}, \nabla u_{3}^{\varepsilon}\right) S^{\prime \prime}\left(u_{3}^{\varepsilon}\right) \nabla u_{3}^{\varepsilon} \\
& \quad=r_{3} \rho_{2}^{\varepsilon}\left(u_{2}^{\varepsilon}\right) S^{\prime}\left(u_{3}^{\varepsilon}\right)-\alpha_{3} u_{3}^{\varepsilon} S^{\prime}\left(u_{3}^{\varepsilon}\right)+h^{\varepsilon} S^{\prime}\left(u_{3}^{\varepsilon}\right) .
\end{aligned}
$$


Use (2.13), (2.14), (H5) - (H7), Lemma 8 and (2.12) with boundedness of $S$ to get

$$
\begin{aligned}
& \frac{\partial S\left(u_{1}\right)}{\partial t}-\operatorname{div}\left(S^{\prime}\left(u_{1}\right) \sigma_{1}\left(u_{2}\right) d_{1}\left(u_{1}, \nabla u_{1}\right)\right)+\sigma_{1}\left(u_{2}\right) d_{1}\left(u_{1}, \nabla u_{1}\right) S^{\prime \prime}\left(u_{1}\right) \nabla u_{1} \\
& \quad=r_{1} \rho_{1}\left(u_{1}\right) S^{\prime}\left(u_{1}\right)-r_{1} u_{2} \mu_{1}\left(u_{1}\right) S^{\prime}\left(u_{1}\right)-\pi\left(u_{1}, u_{3}\right) S^{\prime}\left(u_{1}\right)+f S^{\prime}\left(u_{1}\right), \\
& \frac{\partial S\left(u_{2}\right)}{\partial t}-\operatorname{div}\left(S^{\prime}\left(u_{2}\right) \sigma_{2}\left(u_{1}\right) d_{2}\left(u_{2}, \nabla u_{2}\right)\right)+\sigma_{2}\left(u_{1}\right) d_{2}\left(u_{2}, \nabla u_{2}\right) S^{\prime \prime}\left(u_{2}\right) \nabla u_{2} \\
& \quad=r_{2} \rho_{2}\left(u_{2}\right) S^{\prime}\left(u_{2}\right)-r_{2} u_{1} \mu_{2}\left(u_{2}\right) S^{\prime}\left(u_{2}\right)+g S^{\prime}\left(u_{2}\right), \\
& \frac{\partial S\left(u_{3}\right)}{\partial t}-\sigma_{3} \operatorname{div}\left(S^{\prime}\left(u_{3}\right) d_{3}\left(u_{3}, \nabla u_{3}\right)\right)+\sigma_{3} d_{3}\left(u_{3}, \nabla u_{3}\right) S^{\prime \prime}\left(u_{3}\right) \nabla u_{3} \\
& \quad=r_{3} \rho_{2}\left(u_{2}\right) S^{\prime}\left(u_{3}\right)-\alpha_{3} u_{3} S^{\prime}\left(u_{3}\right)+h S^{\prime}\left(u_{3}\right) .
\end{aligned}
$$

as $\varepsilon$ tends to 0 . By Lemma 2 and Aubin type of lemma one can easily find that $S\left(u_{i}^{\varepsilon}(x, 0)\right)=S\left(u_{i, 0}^{\varepsilon}(x)\right)$ converges to $S\left(u_{i, 0}\right)$ strongly in $H^{-1, s}(\Omega)$, where $s<$ $\inf \left(2, \frac{N}{N-1}\right)$. Then (H4), (H6) and smoothness of $S$ prove the strong convergence in $L^{2}(\Omega)$. Hence, we conclude that $S\left(u_{i}(x, 0)\right)=S\left(u_{i, 0}(x)\right)$. From the above results, we conclude the existence of renormalized solutions of system (1.1).

\section{Entropy solutions for cancer invasion system}

In this section, we have established the second main result of the paper, that is, the renormalized solution is also an entropy solution.

DeFinition 4. An entropy solution (1.1) is a triple $\left(u_{1}, u_{2}, u_{3}\right)$ satisfying the following conditions: for $i=1,2,3, u_{i} \in L^{\infty}\left(0, T ; L^{1}(\Omega)\right) \cap C\left([0, T], L^{1}(\Omega)\right)$. For any $k>0$ and for all $\phi_{i} \in C^{1}\left(Q_{T}\right)$ with $\phi_{i}=0$ in $\Sigma_{T}$,

$$
\begin{aligned}
\int_{\Omega} \tilde{T}_{k}\left(u_{1}\right. & \left.-\phi_{1}\right)(T) \mathrm{d} x-\int_{\Omega} \tilde{T}_{k}\left(u_{1}-\phi_{1}\right)(0) \mathrm{d} x+\int_{0}^{T}\left\langle\phi_{1 t}, T_{k}\left(u_{1}-\phi_{1}\right)\right\rangle \mathrm{d} t \\
& +\int_{Q_{T}} \sigma_{1}\left(u_{2}\right) d_{1}\left(u_{1}, \nabla u_{1}\right) \nabla T_{k}\left(u_{1}-\phi_{1}\right) \mathrm{d} x \mathrm{~d} t \\
& =\int_{Q_{T}}\left(r_{1} \rho_{1}\left(u_{1}\right)-r_{1} u_{2} \mu_{1}\left(u_{1}\right)-\pi\left(u_{1}, u_{3}\right)+f\right) T_{k}\left(u_{1}-\phi_{1}\right) \mathrm{d} x \mathrm{~d} t \\
\int_{\Omega} \tilde{T}_{k}\left(u_{2}-\right. & \left.\phi_{2}\right)(T) \mathrm{d} x-\int_{\Omega} \tilde{T}_{k}\left(u_{2}-\phi_{2}\right)(0) \mathrm{d} x+\int_{0}^{T}\left\langle\phi_{2 t}, T_{k}\left(u_{2}-\phi_{2}\right)\right\rangle \mathrm{d} t \\
& +\int_{Q_{T}} \sigma_{2}\left(u_{1}\right) d_{2}\left(u_{2}, \nabla u_{2}\right) \nabla T_{k}\left(u_{2}-\phi_{2}\right) \mathrm{d} x \mathrm{~d} t \\
& =\int_{Q_{T}}\left(r_{2} \rho_{2}\left(u_{2}\right)-r_{2} u_{1} \mu_{2}\left(u_{2}\right)+g\right) T_{k}\left(u_{1}-\phi_{1}\right) \mathrm{d} x \mathrm{~d} t \\
& +\phi_{Q_{T}} \sigma_{3} d_{3}\left(u_{3}, \nabla u_{3}\right) \nabla T_{k}\left(u_{3}-\phi_{3}\right) \mathrm{d} x \mathrm{~d} t
\end{aligned}
$$




$$
=\int_{Q_{T}}\left(r_{3} \rho_{2}\left(u_{2}\right)-\alpha_{3} u_{3}+h\right) T_{k}\left(u_{3}-\phi_{3}\right) \mathrm{d} x \mathrm{~d} t
$$

hold.

Theorem 2. Under the hypotheses $(H 1)-(H 5)$, the renormalized solution of system (1.1) is also an entropy solution in the sense of Definition 4.

Proof. For $i=1,2,3$, we use $T_{k}\left(u_{i}^{\varepsilon}-\phi_{i}\right)$ as the test functions respectively in the equations $(2.1)$ and for $k>0, \phi_{i} \in C^{1}\left(\bar{Q}_{T}\right)$ with $\phi_{i}=0$ in $\Sigma_{T}$. Multiply (2.1) by $T_{k}\left(u_{i}^{\varepsilon}-\phi_{i}\right)$ respectively and integrating over $Q_{T}$, to get

$$
\begin{gathered}
\int_{0}^{T}\left\langle u_{1 t}^{\varepsilon}, T_{k}\left(u_{1}^{\varepsilon}-\phi_{1}\right)\right\rangle \mathrm{d} t+\int_{Q_{T}} \sigma_{1}\left(u_{2}^{\varepsilon}\right) d_{1}\left(u_{1}^{\varepsilon}, \nabla u_{1}^{\varepsilon}\right) \nabla T_{k}\left(u_{1}^{\varepsilon}-\phi_{1}\right) \mathrm{d} x \mathrm{~d} t \\
=\int_{Q_{T}}\left(r_{1} \rho_{1}^{\varepsilon}\left(u_{1}^{\varepsilon}\right)-r_{1} u_{2}^{\varepsilon} \mu_{1}^{\varepsilon}\left(u_{1}^{\varepsilon}\right)-\pi^{\varepsilon}\left(u_{1}^{\varepsilon}, u_{3}^{\varepsilon}\right)+f^{\varepsilon}\right) T_{k}\left(u_{1}^{\varepsilon}-\phi_{1}\right) \mathrm{d} x \mathrm{~d} t \\
\int_{0}^{T}\left\langle u_{2 t}^{\varepsilon}, T_{k}\left(u_{2}^{\varepsilon}-\phi_{2}\right)\right\rangle \mathrm{d} t+\int_{Q_{T}} \sigma_{2}\left(u_{1}^{\varepsilon}\right) d_{2}\left(u_{2}^{\varepsilon}, \nabla u_{2}^{\varepsilon}\right) \nabla T_{k}\left(u_{2}^{\varepsilon}-\phi_{2}\right) \mathrm{d} x \mathrm{~d} t \\
=\int_{Q_{T}}\left(r_{2} \rho_{2}^{\varepsilon}\left(u_{2}^{\varepsilon}\right)-r_{2} u_{1}^{\varepsilon} \mu_{2}^{\varepsilon}\left(u_{2}^{\varepsilon}\right)+g^{\varepsilon}\right) T_{k}\left(u_{2}^{\varepsilon}-\phi_{2}\right) \mathrm{d} x \mathrm{~d} t \\
\int_{0}^{T}\left\langle u_{3 t}^{\varepsilon}, T_{k}\left(u_{3}^{\varepsilon}-\phi_{3}\right)\right\rangle \mathrm{d} t+\sigma_{3} \int_{Q_{T}} d_{3}\left(u_{3}^{\varepsilon}, \nabla u_{3}^{\varepsilon}\right) \nabla T_{k}\left(u_{3}^{\varepsilon}-\phi_{3}\right) \mathrm{d} x \mathrm{~d} t \\
=\int_{Q_{T}}\left(r_{3} \rho_{2}^{\varepsilon}\left(u_{2}^{\varepsilon}\right)-\alpha_{3} u_{3}^{\varepsilon}+h^{\varepsilon}\right) T_{k}\left(u_{3}^{\varepsilon}-\phi_{3}\right) \mathrm{d} x \mathrm{~d} t .
\end{gathered}
$$

Choose $L=k+\left\|\phi_{1}\right\|_{L^{\infty}\left(Q_{T}\right)}$ and $u_{1 t}^{\varepsilon}=\left(u_{1}^{\varepsilon}-\phi_{1}\right)_{t}-\phi_{1 t}$, we get

$$
\begin{aligned}
& \int_{Q_{T}} d_{1}\left(u_{1}^{\varepsilon}, \nabla u_{1}^{\varepsilon}\right) \nabla T_{k}\left(u_{1}^{\varepsilon}-\phi_{1}\right) \mathrm{d} x \mathrm{~d} t \\
& \quad=\int_{Q_{T}} d_{1}\left(T_{L}\left(u_{1}^{\epsilon}\right), \nabla T_{L}\left(u_{1}^{\epsilon}\right)\right) \nabla T_{k}\left(u_{1}^{\epsilon}\right)\left(T_{L}\left(u_{1}^{\epsilon}\right)-\phi_{1}\right) \mathrm{d} x \mathrm{~d} t, \\
& \int_{0}^{T}\left\langle u_{1 t}^{\varepsilon}, T_{k}\left(u_{1}^{\varepsilon}-\phi_{1}\right)\right\rangle \mathrm{d} t=\int_{\Omega} \tilde{T}_{k}\left(u_{1}^{\varepsilon}-\phi_{1}\right)(T) \mathrm{d} x-\int_{\Omega} \tilde{T}_{k}\left(u_{1}^{\varepsilon}-\phi_{1}\right)(0) \mathrm{d} x \\
& \quad+\int_{0}^{T}\left\langle\phi_{1 t}, T_{k}\left(T_{L}\left(u_{1}^{\varepsilon}\right)-\phi_{1}\right)\right\rangle \mathrm{d} t .
\end{aligned}
$$

From the above equations, we get

$$
\begin{aligned}
\int_{\Omega} \tilde{T}_{k}\left(u_{1}^{\varepsilon}\right. & \left.-\phi_{1}\right)(T) \mathrm{d} x-\int_{\Omega} \tilde{T}_{k}\left(u_{1}^{\varepsilon}-\phi_{1}\right)(0) \mathrm{d} x+\int_{0}^{T}\left\langle\phi_{1 t}, T_{k}\left(T_{L}\left(u_{1}^{\varepsilon}\right)-\phi_{1}\right)\right\rangle \mathrm{d} t \\
& +\int_{Q_{T}} \sigma_{1}\left(u_{2}^{\varepsilon}\right) d_{1}\left(T_{L}\left(u_{1}^{\varepsilon}\right), \nabla T_{L}\left(u_{1}^{\varepsilon}\right)\right) \nabla T_{k}\left(T_{L}\left(u_{1}^{\varepsilon}\right)-\phi_{1}\right) \mathrm{d} x \mathrm{~d} t \\
& =\int_{Q_{T}}\left(r_{1} \rho_{1}^{\varepsilon}\left(u_{1}^{\varepsilon}\right)-r_{1} u_{2}^{\varepsilon} \mu_{1}^{\varepsilon}\left(u_{1}^{\varepsilon}\right)-\pi^{\varepsilon}\left(u_{1}^{\varepsilon}, u_{3}^{\varepsilon}\right)+f^{\varepsilon}\right) T_{k}\left(u_{1}^{\varepsilon}-\phi_{1}\right) \mathrm{d} x \mathrm{~d} t
\end{aligned}
$$


Since $\tilde{T}_{k}$ is Lipschitz continuous, using (2.13), we have

$$
\begin{aligned}
& \int_{\Omega} \tilde{T}_{k}\left(u_{1}^{\varepsilon}-\phi_{1}\right)(T) \mathrm{d} x \rightarrow \int_{\Omega} \tilde{T}_{k}\left(u_{1}-\phi_{1}\right)(T) \mathrm{d} x, \\
& \int_{\Omega} \tilde{T}_{k}\left(u_{1}^{\varepsilon}-\phi_{1}\right)(0) \mathrm{d} x \rightarrow \int_{\Omega} \tilde{T}_{k}\left(u_{1}-\phi_{1}\right)(0) \mathrm{d} x, \text { as } \varepsilon \rightarrow 0 .
\end{aligned}
$$

Using the strong convergence of $f^{\varepsilon},(2.13),(2.14)$ and the Lemma 8 as $\varepsilon \rightarrow 0$, we get

$$
\begin{aligned}
& \int_{\Omega} \tilde{T}_{k}\left(u_{1}-\phi_{1}\right)(T) \mathrm{d} x-\int_{\Omega} \tilde{T}_{k}\left(u_{1}-\phi_{1}\right)(0) \mathrm{d} x+\int_{0}^{T}\left\langle\phi_{1 t}, T_{k}\left(T_{L}\left(u_{1}\right)-\phi_{1}\right)\right\rangle \mathrm{d} t \\
& \quad+\int_{Q_{T}} \sigma_{1}\left(u_{2}\right) d_{1}\left(u_{1}, \nabla u_{1}\right) \nabla T_{k}\left(u_{1}-\phi_{1}\right) \mathrm{d} x \mathrm{~d} t \\
& \quad=\int_{Q_{T}}\left(r_{1} \rho_{1}\left(u_{1}\right)-r_{1} u_{2} \mu_{1}\left(u_{1}\right)-\pi\left(u_{1}, u_{3}\right)+f\right) T_{k}\left(u_{1}-\phi_{1}\right) \mathrm{d} x \mathrm{~d} t
\end{aligned}
$$

Similarly, we get

$$
\begin{aligned}
& \int_{\Omega} \tilde{T}_{k}\left(u_{2}-\phi_{2}\right)(T) \mathrm{d} x-\int_{\Omega} \tilde{T}_{k}\left(u_{2}-\phi_{2}\right)(0) \mathrm{d} x+\int_{0}^{T}\left\langle\phi_{2 t}, T_{k}\left(u_{2}-\phi_{2}\right)\right\rangle \mathrm{d} t \\
& \quad+\int_{Q_{T}} \sigma_{2}\left(u_{1}\right) d_{2}\left(u_{2}, \nabla u_{2}\right) \nabla T_{k}\left(u_{2}-\phi_{2}\right) \mathrm{d} x \mathrm{~d} t \\
& =\int_{Q_{T}}\left(r_{2} \rho_{2}\left(u_{2}\right)-r_{2} u_{1} \mu_{2}\left(u_{2}\right)+g\right) T_{k}\left(u_{2}-\phi_{2}\right) \mathrm{d} x \mathrm{~d} t, \\
& \int_{\Omega} \tilde{T}_{k}\left(u_{3}-\phi_{3}\right)(T) \mathrm{d} x-\int_{\Omega} \tilde{T}_{k}\left(u_{3}-\phi_{3}\right)(0) \mathrm{d} x+\int_{0}^{T}\left\langle\phi_{3 t}, T_{k}\left(u_{3}-\phi_{3}\right)\right\rangle \mathrm{d} t \\
& \quad+\sigma_{3} \int_{Q_{T}} d_{3}\left(u_{3}, \nabla u_{3}\right) \nabla T_{k}\left(u_{3}-\phi_{3}\right) \mathrm{d} x \mathrm{~d} t \\
& =\int_{Q_{T}}\left(r_{3} \rho_{2}\left(u_{2}\right)-\alpha_{3} u_{3}+h\right) T_{k}\left(u_{3}-\phi_{3}\right) \mathrm{d} x \mathrm{~d} t,
\end{aligned}
$$

for all $k>0$ and for $i=1,2,3, \phi_{i} \in C^{1}\left(\bar{Q}_{T}\right)$ with $\phi_{i}=0$ in $\Sigma_{T}$. This completes the proof of existence of entropy solutions of the system (1.1).

\section{Acknowledgement}

We would like to thank Prof. Francesco Petitta, Dipartimento di Scienze di Base e Applicate per IIngegneria, Universit di Roma, Via A. Scarpa, 16 - 00161, Roma for his valuable suggestions to improve the quality of the article. The work of the first author is supported by the DST-SERB Early Career Award File No. ECR/2016/000624. The work of second author is supported by the University Grants Commission F./2015-16/NFO-2015-17-OBC-TAM$46284 /(\mathrm{SA}-\mathrm{III} /$ Website $)$ ) and the work of third author is supported by the DST-SERB YSS/2015/002104. 


\section{References}

[1] K. Ammar. Renormalized entropy solutions for degenerate nonlinear evolution problems. Electron. J. Differential Equations, 147:1-32, 2009.

[2] M. Bendahmane. Weak and classical solutions to predator-prey system with cross-diffusion. Nonlinear Anal., 73(8):2489-2503, 2010. https://doi.org/10.1016/j.na.2010.06.021.

[3] M. Bendahmane and K.H. Karlsen. Renormalized entropy solutions for quasilinear anisotropic degenerate parabolic equations. SIAM J. Math. Anal., 36(2):405-422, 2004. https://doi.org/10.1137/S0036141003428937.

[4] M. Bendahmane and K.H. Karlsen. Analysis of a class of degenerate reactiondiffusion systems and the bidomain model of cardiac tissue. Netw. Heterog. Media., 1(1):185-218, 2006.

[5] M. Bendahmane and K.H. Karlsen. Renormalized solutions of an anisotropic reaction-diffusion-advection systems with $L^{1}$ data modeling the propagation of an epidemic disease. Commun. Pure Appl. Anal., 5(4):733-762, 2006.

[6] A. Bertuzzi, A. Fasano, A. Gandolfi and C. Sinisgalli. Necrotic core in EMT6/Ro tumor spheroids: is it caused by an ATP deficit? J. Theor. Biol., 262(1):142150, 2010. https://doi.org/10.1016/j.jtbi.2009.09.024.

[7] L. Bianchini and A. Fasano. A model combining acid-mediated tumor invasion and nutrient dynamics. Nonlinear Anal. Real World Appl., 10(4):1955-1975, 2009. https://doi.org/10.1016/j.nonrwa.2008.03.001.

[8] D. Blanchard and F. Murat. Renormalised solutions of nonlinear parabolic problems with $L^{1}$ data:existence and uniqueness. Proc. Roy. Soc. Edinburgh Sect. A, 127(6):1137-1152, 1997. https://doi.org/10.1017/S0308210500026986.

[9] D. Blanchard, F. Murat and H. Redwane. Existence and uniqueness of a renormalized solution for a fairly general class of nonlinear parabolic problems. J. Differential Equations, 177(2):331-374, 2001. https://doi.org/10.1006/jdeq.2000.4013.

[10] J. Carrillo and P. Wittbold. Renormalized entropy solutions of scalar conservation laws with boundary condition. J. Differential Equations, 185(1):137-160, 2002. https://doi.org/10.1006/jdeq.2002.4179.

[11] R.J. DiPerna and P.L. Lions. On the Cauchy problem for Boltzmann equations: Global existence and weak stability. Ann. of Math., 130(2):321-366, 1989.

[12] J. Droniou and A. Prignet. Equivalence between entropy and renormalized solutions for parabolic equations with smooth measure data. NoDEA Nonlinear Differential Equations Appl., 14(1-2):181-205, 2007. https://doi.org/10.1007/s00030-007-5018-z.

[13] S. Ganesan and S. Lingeshwaran. A biophysical model of tumor invasion. Commun. Nonlinear Sci. Numer. Simulat., 46:135-152, 2017. https://doi.org/10.1016/j.cnsns.2016.10.013.

[14] R.A. Gatenby and E.T. Gawlinski. A reaction-diffusion model of cancer invasion. Cancer Res., 56(24):5745-5753, 1996.

[15] R.A. Gatenby, E.T. Gawlinski, A.F. Gmitro, B. Kaylor and R.J. Gillies. Acidmediated tumor invasion: a multidisciplinary study. Cancer Res., 66(10):52165223, 2006. https://doi.org/10.1158/0008-5472.CAN-05-4193. 
[16] C. Li, A. Kaushik and G. Yin. Global existence of classical solutions to an acidmediated invasion model for tumor-stromal interactions. Appl. Math. Comput., 234:599-605, 2014. https://doi.org/10.1016/j.amc.2014.02.057.

[17] N.K. Martin, E.A. Gaffney, R. A.Gatenby and P.K. Maini. Tumour-stromal interactions in acid-mediated invasion: a mathematical model. J. Theoret. Biol., 267(3):461-470, 2010. https://doi.org/10.1016/j.jtbi.2010.08.028.

[18] G. Dal Maso, F. Murat, L. Orsina and A. Prignet. Renormalized solutions of elliptic equations with general measure data. Ann. Scuola Norm. Sup. Pisa Cl. Sci., 28(4):741-808, 1999.

[19] J.B. McGillen, E.A. Gaffney, N.K. Martin and P.K. Maini. A general reactiondiffusion model of acidity in cancer invasion. J. Math. Biol., 68(5):1199-1224, 2014. https://doi.org/10.1007/s00285-013-0665-7.

[20] G. Meral, C. Stinner and C. Surulescu. A multiscale model for acid-mediated tumor invasion: therapy approaches. J. Coupled Syst. Multiscale Dyn., 3(2):135142, 2015. https://doi.org/10.1166/jcsmd.2015.1071.

[21] G. Meral and C. Surulescu. Mathematical modelling, analysis and numerical simulations for the influence of heat shock proteins on tumour invasion. J. Math. Anal. Appl., 408(2):597-614, 2013. https://doi.org/10.1016/j.jmaa.2013.06.017.

[22] F. Petitta, A.C. Ponce and A. Porretta. Diffuse measures and nonlinear parabolic equations. J. Evol. Equ., 11(4):861-905, 2011. https://doi.org/10.1007/s00028011-0115-1.

[23] F. Petitta and A. Porretta. On the notion of renormalized solution to nonlinear parabolic equations with general measure data. J. Elliptic Parabol. Equ., 1(1):201-214, 2015. https://doi.org/10.1007/BF03377376.

[24] L. Shangerganesh and K. Balachandran. Renormalized and entropy solutions of nonlinear parabolic system. Electron. J. Differential Equations, 268:1-24, 2013.

[25] L. Shangerganesh, N. Barani Balan and K. Balachandran. Weak-renormalized solutions for predator-prey system. Appl. Anal., 92(3):441-459, 2011. https://doi.org/10.1080/00036811.2011.625014.

[26] K. Smallbone, D.J. Gavaghan, R.A. Gatenby and P.K. Maini. The role of acidity in solid tumour growth and invasion. J. Theoret. Biol., 235(4):476-484, 2005. https://doi.org/10.1016/j.jtbi.2005.02.001.

[27] Y. Tao and J.I. Tello. Nonlinear stability of a heterogeneous state in a PDE-ODE model for acid-mediated tumor invasion. Math. Biosci. Eng., 13(1):193-207, 2016. https://doi.org/10.3934/mbe.2016.13.193.

[28] R. Venkatasubramanian, M.A. Henson and N.S. Forbes. Incorporating energy metabolism into a growth model of multicellular tumor spheroids. J. Theor. Biol., 242(2):440-453, 2006. https://doi.org/10.1016/j.jtbi.2006.03.011. 